\title{
Efficacy Before Novelty: Establishing Design Guidelines in Interactive Gaming for Rehabilitation and Training
}

\author{
by
}

Monica Zaczynski

A thesis submitted to the Faculty of Graduate and Postdoctoral Affairs in partial fulfillment of the requirements for the degree of

\author{
Master of Applied Science \\ in \\ Human-Computer Interaction
}

Carleton University

Ottawa, Ontario

(C) 2013, Monica Zaczynski 


\begin{abstract}
Interactive gaming has demonstrated promise as a low-cost, at-home physiotherapy supplement. Gaming systems offer convenience and the ability to provide enhanced reporting and progress data if body measurement information is collected effectively. Current commercially available systems are not necessarily designed for rehabilitation and as a result, the quality of instruction delivery and level of involvement falls short of the needs of patients.

Many variables contribute to user understanding. This thesis will look at adapting for occlusion and lack of visibility; learning and orientation; and providing feedback in an effort to determine if there is an ideal visual demonstration delivery that maximizes pose understanding and user self-efficacy, determine whether supplementary modalities are important for instruction, and determine if there is an ideal feedback delivery that promotes pose comprehension, confidence and motivation. This information can provide a guideline for designing clear and supportive, interactive training or rehabilitation systems that can engage users, prevent injury and help maintain fitness.
\end{abstract}




\section{Acknowledgements}

Thank you to those who inspired me, humoured me, reassured me, listened when I went on tangents. Thank you to those who pointed me in all the right directions when I didn't have one. Thank you to those who made this a big deal - to keep me motivated and those who reminded me it's not a big deal - to keep me sane. Thank you to those who jumped through virtual hoops for me, cooked for me when I couldn't spare the time and called me regularly to make sure I was alive, well and still on track. Thank you. 


\section{Table of Contents}

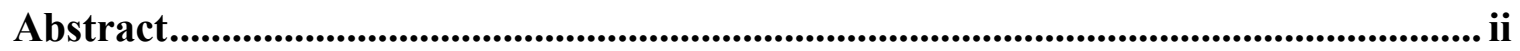

Acknowledgements ............................................................................................................................. iii

Table of Contents ........................................................................................................ iv

List of Tables ............................................................................................................................... vii

List of Figures.............................................................................................................................. viii

List of Appendices........................................................................................................................... ix

1. Introduction ........................................................................................................................ 1

1.1 Overview and motivation ...................................................................................

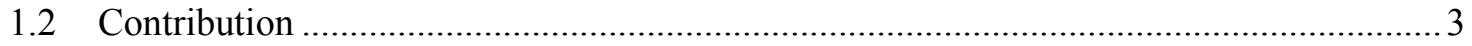

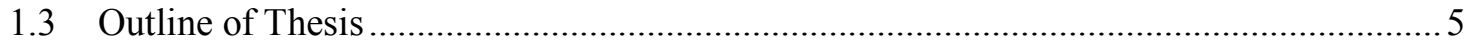

2. Related Works....................................................................................................................... 6

2.1 A Case For Alternate Methods Of Physiotherapy ........................................................... 6

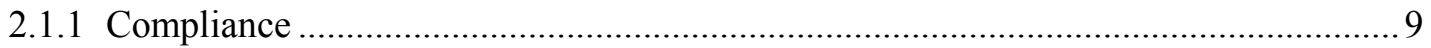

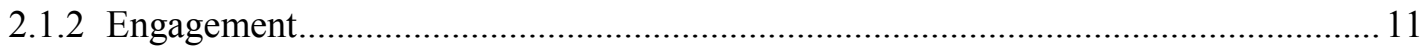

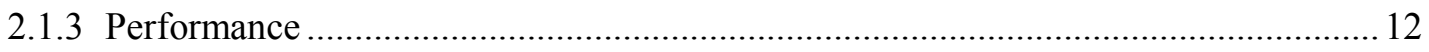

2.2 Interactive Gaming For At-Home Physiotherapy …………………………………..... 13

2.2.1 Body Measuring Machine ............................................................................................ 14

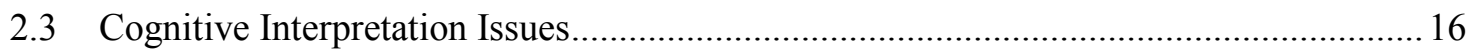

2.3.1.1 Adapting For Occlusion.................................................................................. 17

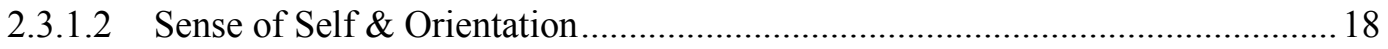

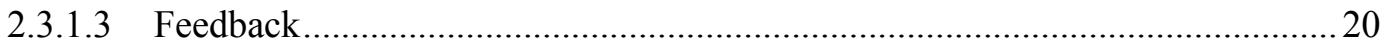

3. Test 1 Demonstration Delivery ................................................................................. 23

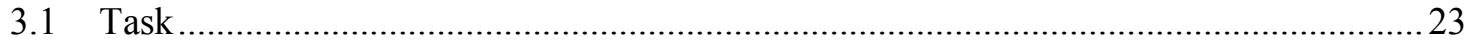




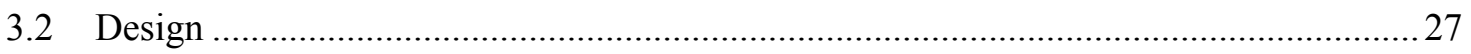

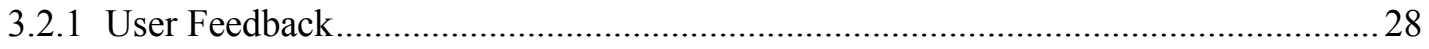

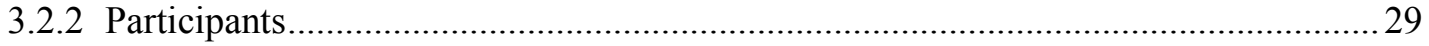

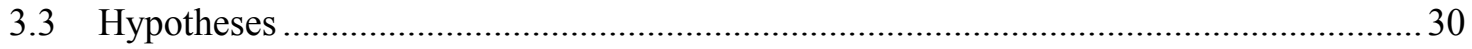

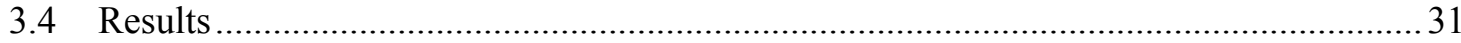

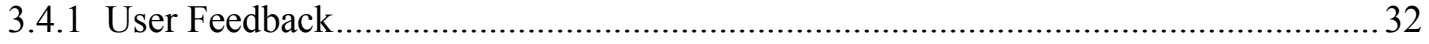

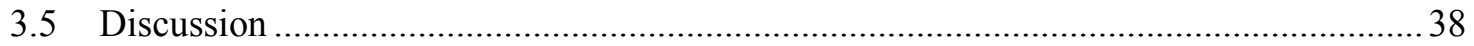

4. Test 2 - Feedback ........................................................................................................ 42

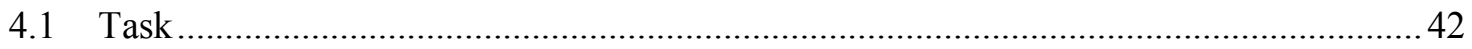

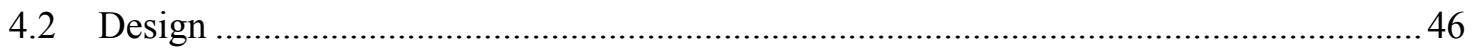

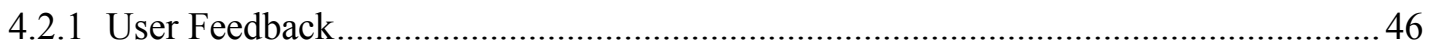

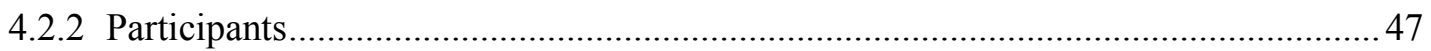

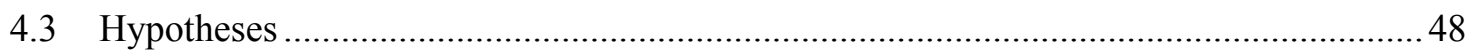

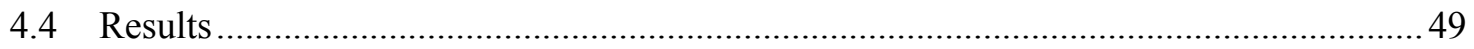

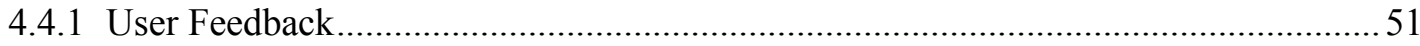

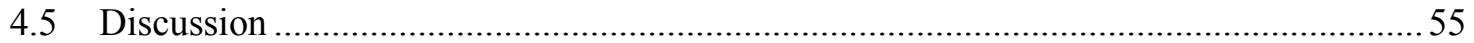

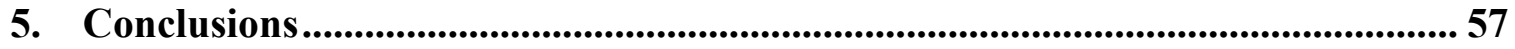

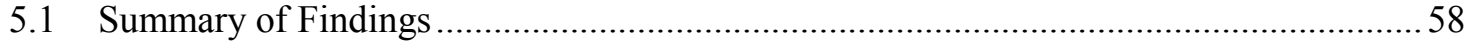

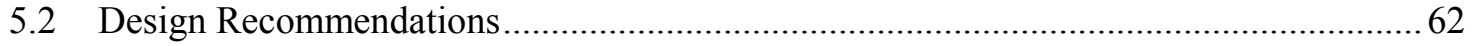

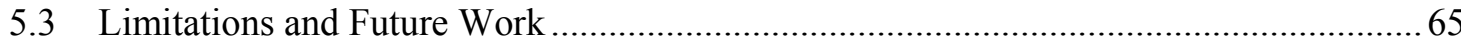

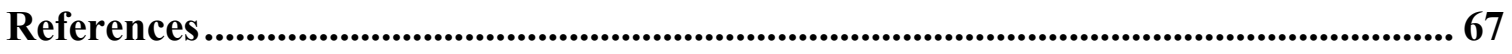

Appendices............................................................................................................................ 74

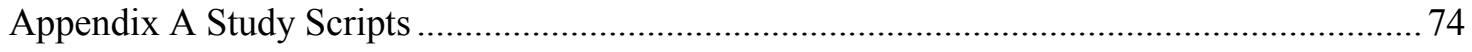

A.1 Demonstration Delivery Test Script ........................................................................ 74

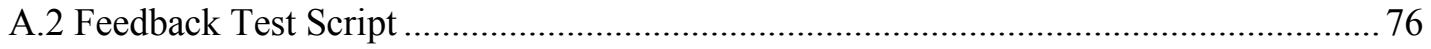




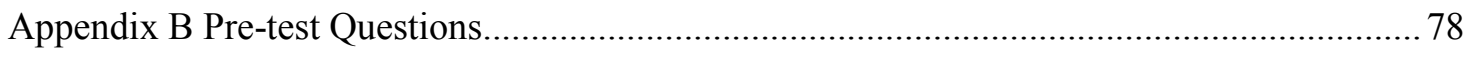

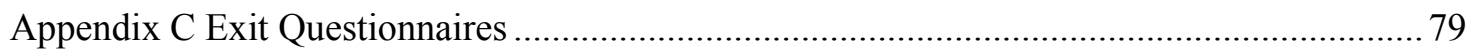

C.1 Demonstration Delivery Test Exit Questionnaire...................................................... 79

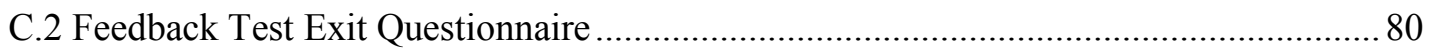

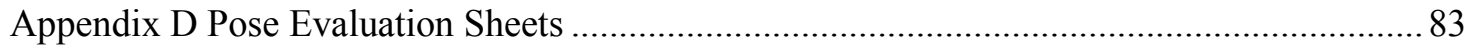

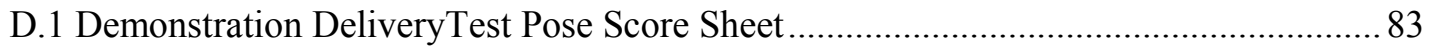

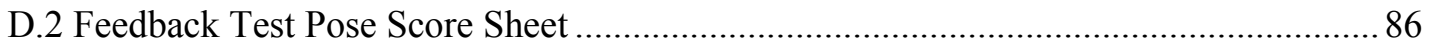




\section{List of Tables}

Table 1 Demonstration - Frequency of instructional class attendance of males and

females 28

Table 2 Demonstration - Distribution of instructional class type for males and females 28

Table 3 Demonstration - Post-pose Likert responses 32

Table 4 Demonstration - Visual responses coded tally ........................................... 34

Table 5 Demonstration - Verbal responses coded tally.......................................... 35

Table 6 Categorical Tally of Comments on Verbal Conditions ..................................... 36

Table 7 Feedback - Distribution of instructional class type for males and females........ 46

Table 8 Feedback - Frequency of instructional class attendance of males and females .. 47

Table 9 Feedback - Mean and Standard Deviation of Likert post-pose responses .......... 49

Table 10 Feedback - Mean and Standard Deviation of exit questionnaire responses...... 52

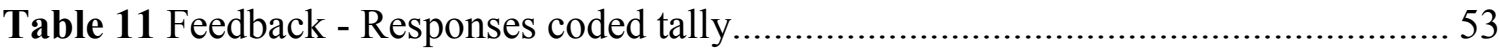




\section{List of Figures}

Figure 1 Side and Front View Demonstrating Occlusion.................................................. 17

Figure 2 Correct Vs. Incorrect Knee Raises .................................................................. 20

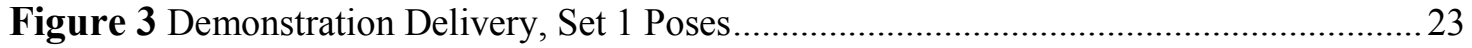

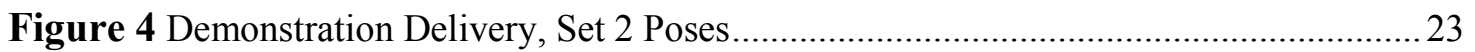

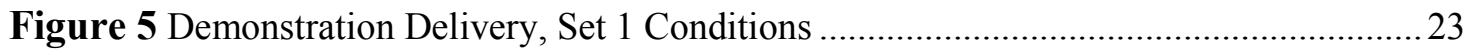

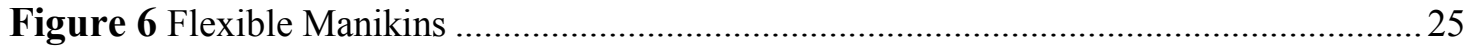

Figure 7 Mean Pose Scores (\%) for Visual Delivery Conditions....................................... 30

Figure 8 Mean Pose Scores (\%) for Verbal Delivery Conditions .......................................... 31

Figure 9 Mean Likert Scores for Quality of Demonstration of visual Delivery Conditions ... 32

Figure 10 Grouped Negative \& Positive Comments for Visual Conditions .......................... 36

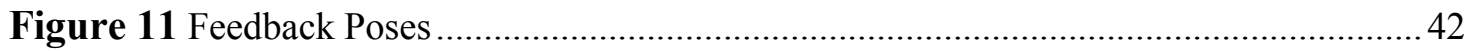

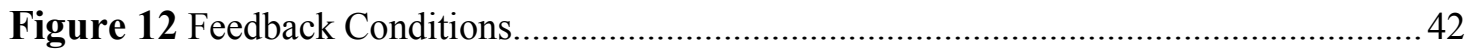

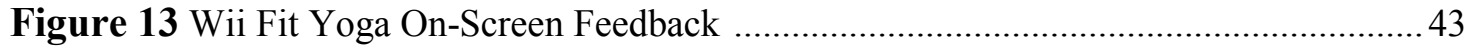

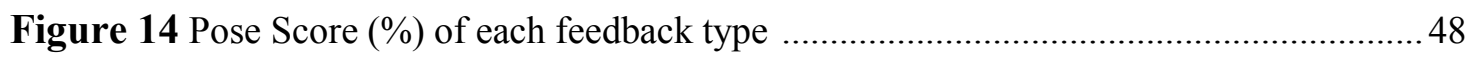

Figure 15 Mean Likert Scores for confidence after feedback ............................................50

Figure 16 Grouped Negative \& Positive Comments for Visual Conditions ...........................51 


\section{List of Appendices}

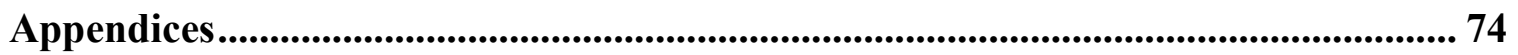

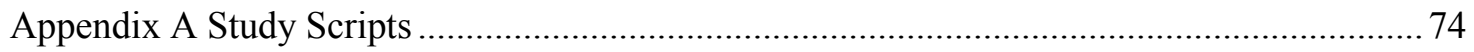

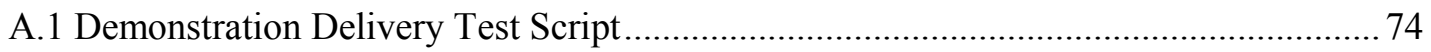

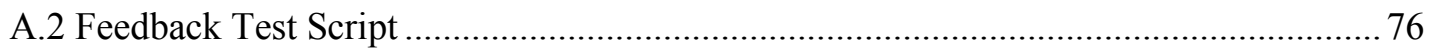

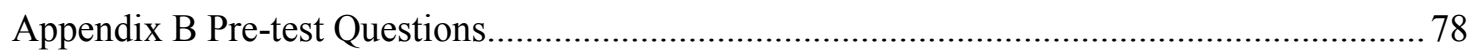

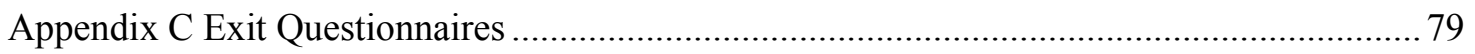

C.1 Demonstration Delivery Test Exit Questionnaire...................................................... 79

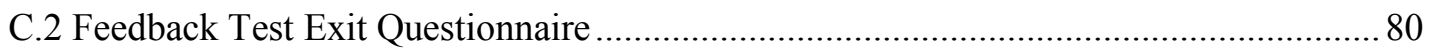

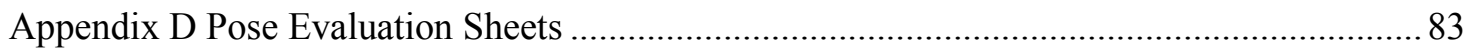

D.1 Demonstration DeliveryTest Pose Score Sheet ....................................................... 83

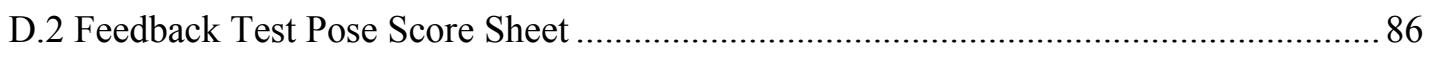




\section{Introduction}

\subsection{Overview and motivation}

Rehabilitating a sport related injury such as a pulled or torn knee ligament typically necessitates several physiotherapy sessions and months of daily independent strengthening exercise. While one-on-one instruction with a physiotherapist offers the most attentive demonstration and feedback, studies have shown that adherence to independent physiotherapy exercise is low $[20,70]$. Without seeing recognizable improvement or receiving any feedback, independent low intensity exercises just don't generate engagement. Physiotherapists often recommend doing a regimen of independent exercises in addition to a low intensity strengthening activity, such as yoga [80]. This offers an alternative that can be performed with others, which has been shown to increase exercise commitment $[12,25,43,81]$. Though it does not provide the same level of feedback that one-on-one instruction does, it provides the engagement and moderate feedback independent exercise lacks.

Traditional in-person rehabilitation and pose-based activities such as yoga have benefitted from offering tailored instruction to participants. Ideally, instructors will demonstrate the pose in a way they determine is beneficial, allow for the participant to attempt, then provide feedback visually, verbally or physically to ensure the pose is executed properly and without injury. With convenient and highly popular interactive gaming systems like Wii and Kinect having released pose based activities like yoga, there seems to be a shift from purely entertainment-based games to fitness based games. Though these systems can provide accurate body mapping and action measurement they 
have been primarily used as entertainment systems; consequently, the question of how effective and clear they can provide precise instruction has not yet been explored.

In the last decade interactive gaming systems such as Nintendo Wii and Xbox Kinect have released yoga and other personal training games that boast a devoted instructor from the comfort of home. These systems have offered an alternative to the traditional fitness facility, which can be expensive, overcrowded and uncomfortable. Through the purchase of a single gaming system, an at home experience is created that can be adapted to the user's preferences. Most games do an adequate job trying to replicate environmental elements of exercise instruction but the social experience of large class instruction and the customized attention of one-on-one instruction is lost through the medium.

In academia, research and prototype software and hardware have been developed $[29,61,76]$ to address the growing need for an alternative physiotherapy solution, however, few to none have reached commercial success. Conversely, the potential limitation of the available commercial games and gaming systems, even though they encourage improvements in balance and strength, is that they are not necessarily designed for rehabilitation [4]. This is, in part, due to the fact that they cannot be adapted for specific patient limitations [33,71] and they lack correction of compensation [28].

There appears to be a need to step back and reassess the requirements of patients. While a great portion of research in the area has focused on refining the system's body measuring technology $[29,61,76]$ or searching for an appropriate blend of entertainment and following the doctor's orders $[9,24,74]$, it seems the quality of the activity execution has not been given due attention [4,24], likewise, the patient's self-efficacy associated 
with fully understanding what is expected. As much as a refined body measuring system and engaging game are important, these needs are essential to ensuring the level of interest and motivation that keep patients motivated long-term $[13,67]$.

Many variables contribute to user understanding. While interactive systems address concerns regarding novelty and convenience, new challenges involving 3D virtual space, visio-spatial understanding and learning must be examined.

\subsection{Contribution}

Literature on compliance reports over 200 factors that may contribute to patient's noncompliance with a physiotherapy regimen including convenience, the nature and level of physiotherapist involvement, and instruction delivery by the physiotherapist $[13,70]$. Interactive gaming has demonstrated promise as a low-cost, at-home physiotherapy supplement $[61,82]$. Gaming systems offer convenience and the ability to provide enhanced reporting and progress data if body measurement information is collected effectively. Current commercially available systems are not necessarily designed for rehabilitation and as a result, the quality of instruction delivery and level of involvement falls short for the needs of patients $[47,77]$.

The aim of this thesis is to determine if there is an ideal visual demonstration delivery that maximizes pose understanding and user self-efficacy, determine whether supplementary modalities are important for instruction, and determine if there is an ideal feedback delivery that promotes pose comprehension, confidence and motivation.

We intend to explore user understanding of pose presentation in low paced rehabilitation or interactive training activities. Many variables contribute to user 
understanding; this study will look at adapting for occlusion and lack of visibility, learning and orientation, adapting for game system capabilities, and providing feedback.

These factors are evaluated in two studies. During the demonstration delivery study, participants are tested on their understanding of yoga poses delivered by a 3D animated instructor meant to replicate the visual style of a video game avatar. Multiple modalities are addressed to obtain a rich understanding of how interactive gaming can assist learning for the benefit of physiotherapy. Here, the orientation is challenged and the amount of occlusion is manipulated using different angles and types of visual delivery. In a second set of poses, the importance of verbal delivery on learning is examined.

While comprehension of the demonstration is one aspect, the second study examines feedback and corrective direction. Many gaming systems offer poor to no feedback once they have demonstrated an activity. At best they inform the user of incorrect placement but rarely tell the user how to correct. Users may not even realize they are performing the activity incorrectly putting them at risk for injury. This study evaluates the impact of feedback delivered using various modalities on learning and pose accuracy.

This thesis builds a patient experience guideline that addresses foundational elements of learning and comprehension, regardless of injury type, in an effort to improve the quality of physiotherapy performance. As a result, this information can provide a guideline for designing clear and supportive, at-home interactive training or rehabilitation systems that can engage users, prevent injury and help maintain fitness. 


\subsection{Outline of Thesis}

This thesis is presented in six chapters. The first chapter introduces the topic of interactive gaming systems as a viable solution to support at-home physiotherapy and low-paced training. We begin by demonstrating the growing need for support in the field of physiotherapy, particularly for less debilitating injuries and conditions.

The second chapter presents a review of related literature beginning with examining the needs of an injured patient. We review the importance of compliance, engagement, and patient comprehension. We then move on to the work already done on interactive gaming in the area of physiotherapy and the challenges being faced, including the body-measuring machine and the interpretation issues associated with a virtual 3D environment.

The third chapter provides detail about our first experiment on demonstration delivery, where participants posed manikins after observing several yoga pose demonstration. This chapter includes descriptions of the task, experimental design, hypotheses, measurements, and the participants. A discussion of the results is also presented in this chapter.

The fourth chapter provides detail about our second experiment on feedback, where participants received different types of feedback while performing a set of yoga poses. This chapter, like the previous, includes description of the task, experimental design, hypotheses, measurements, and a discussion of the results.

The fifth chapter summarizes the main findings of the thesis, provides a set of design recommendations, and describes the limitations and future work in this area. 


\section{Related Works}

\subsection{A Case For Alternate Methods Of Physiotherapy}

In light of the aging population, the projected increase in government spending on health and increased public awareness of health care, Service Canada has reported that the need for physiotherapists will continue to increase sharply over the next few years [65]. National shortages of physiotherapists in Canada have resulted in virtually no unemployment in the field. Immigration Canada has declared physiotherapy one of 29 high demand jobs [10] and with lower than average numbers nearing retirement and future opportunities limited to new graduates of competitive training programs, demand is slow to meet the need [65]. Alternative methods of administering comprehensive physiotherapy treatment to less debilitating injuries are needed to reduce the stress on demand.

Interactive video games can offer an engaging alternative to paper print-out athome exercise routines. They are commercially available and relatively inexpensive compared to a dedicated physiotherapist or other assistive rehabilitation technology [8]. Additionally, they can provide feedback in varying modalities and progress tracking with the assistance of body movement capture technologies [11,28]. Exploration using interactive video game systems has demonstrated promising results as a supporting element to specific recovery routines $[61,77,82]$.

Like each injury, each road to recovery will be different. Motivations and interest will vary with injury severity and even each person. While more debilitating injuries generate greater motivation to follow a rehabilitation routine in order to return to normal function [27,69], a typical sport or occupational injury is mainly tended to while the 
discomfort is greatest. Noticeable improvement can also preserve motivation. This can be observed in stroke recovery patients [27]. The loss or impairment of gross motor skills means that almost any activities can advance recovery. Conversely, observing improvement in a sprained ankle may be noticeable at first but once relatively normal function has returned, motivation to continue strengthening activities often decreases.

The flexibility of commercial interactive gaming systems have allowed them to be used for various kinds of rehabilitation including emotional trauma [47], gaining gross motor function back post-stroke $[2,11,26,35,48,82,83]$, building strength after a bone or muscle injury [18,36,51], seniors mobility [1,6,30,31,42], and cerebral palsy [19]. Gaming systems can offer additional flexible, customizable, at-home monitoring and motivation to a patient's regular rehabilitation visits [75] .

Patients with musculoskeletal injury, those who suffered a bone or muscle/ligament injury, are typically younger and more numerous than other patients needing rehabilitation. According to eHealthMD, anterior cruciate ligament (ACL) ruptures occur at a rate of 60 per 100,000 people per year [22] and the American Association of Orthopedic Surgeons reported that 25,000 individuals sprain their ankle every day [3]. Physiotherapy is often required for musculoskeletal injuries to help return to normal function and reduce the likelihood of re-injury [17]. Due to the high number of musculoskeletal injuries and relatively low severity, the cost and time associated with one-on-one physiotherapy is simply too high to warrant abundant professional time, as a result, unsupervised, at-home activities are often prescribed.

As an example, soccer players are frequent victims of knee, especially ACL, injuries. Sharp stops and changes of direction, which routinely occur in-game, are the 
leading cause of ACL damage. An ACL injury would cause considerable swelling, reduction of range of movement and pain while walking within hours of injury. Reconstructive surgery has become a common procedure when the ACL is fully torn, requiring several weeks of physiotherapy, while a partial tear may simply require some icing and regular physiotherapy for several weeks to return to normal function. With good physiotherapy, $90-95 \%$ of patients return to their pre-injury sports participation within six months [22].

Today, physiotherapists use a combination of practices to remedy swelling, scar tissue, and muscle degradation such as acupuncture, ultrasound and electro-stimulation in addition to a regimen of at-home exercises to rebuild muscle strength [14]. Physiotherapists typically prescribe a set of between 4 and 7 exercises for the patient to perform at home daily until the next visit. Once the physiotherapist demonstrates the exercises, the patient will perform a full set of the routine while at the clinic so the physiotherapist can provide feedback and ensure the patient understands the exercise. However, by bringing the therapy out of the clinic and into the patient's home, the benefit of dedicated supervision is lost. This often results in exercises being performed incorrectly [4] or the regimen not being performed at all $[34,68]$. The cause of the former situation is typically that a well-meaning patient cannot remember the proper form, execution, or order. Elements and subtleties of exercises are often forgotten and without supervision patients do not receive any oversight on the quality or accuracy of their performance $[40,72]$.

As such a gaming system can assist with these memory issues. While gaming systems are widely used for various types of rehabilitation to promote engagement, 
fundamental problems with visual presentation, understanding and feedback (in general as well as with on-screen avatars), have become more evident with increasing need for measurement and activity precision. Each of these elements will be discussed below.

\subsubsection{Compliance}

A patient with a sports-related injury may have many reasons for not continuing with prescribed at-home exercises. Literature on compliance reports over 200 factors that may contribute to patient's noncompliance with a physiotherapy regimen. Reports on compliance suggest that one-third to two thirds of patients are found to be noncompliant with exercise $[21,37,38,40,50]$. Physical therapists themselves estimate that $64 \%$ of their patients comply with short-term exercise regimens, but that only $23 \%$ of them persevere with exercising in the long run [68]. Whether these estimates are realistic or not is unknown, but it is difficult to assess patients' compliance because patients often do not admit noncompliance [34]. Some of the consistent factors that contribute to noncompliance include convenience, the nature and level of physiotherapist involvement and also the instruction delivery by the physiotherapist, each of which will be discussed further.

Convenience was found to be an important factor in predicting patient compliance [21,34]. For this reason, physiotherapists often recommend a combination of practices such as class-based activities like Yoga that are flexible for the patient's schedule, can assist with strengthening, and keep a patient interested in the rehabilitation process [5]. This offers an alternative that can be performed with others, which has been shown to increase exercise commitment $[12,25,43,81]$. However, the amount of devoted attention 
from the instructor is relative to the number of people in the class. Risk of injury may increase due to lack of attentive supervision.

Gaming systems such as Nintendo Wii and Xbox Kinect have released yoga and other personal training games $[59,79]$ that boast a devoted instructor from the comfort of home. These systems compensate for that lack of dedicated attention while addressing the high cost, and overcrowded group settings of gyms, which may be deterrents from participating for some people.

Similarly, the attention paid by the physiotherapist and amount of involvement in the recovery process has also demonstrated a positive effect on compliance $[21,23,34]$. Patients who received ongoing feedback about their compliance, progress and efforts are more dedicated than patients without supervision $[23,34]$. Oldridge reports that adherence dropped from $59 \%$ in the supervised program to $29 \%$ after 6 months of unsupervised exercise [53:470]. Having the physiotherapist offer feedback during exercise can increase feelings of self-efficacy and confidence in the patient's meaningful progress towards recovery $[39,54,67]$. This dedicated involvement can also lead to increased patient accountability for actions and progress. While this is not possible when patients perform exercises out of clinic, gaming systems can support physiotherapist involvement between clinic visits by offering precise feedback on exercises. Additionally, they can provide short and long-term progress monitoring by the patient's physiotherapist remotely [44].

While the quantity of meaningful involvement is important, the quality also impacts progress. The delivery of instruction is an important element in patient compliance. When physiotherapists deliver unclear instruction and fail to explain the 
rationale for the instructions, unintended noncompliance can arise from forgetting or misinterpretation [40,72]. A well-meaning patient can be discouraged or even become injured further as a result. While cognitive and learning issues are considered when delivering instruction to a patient, the use of a gaming system introduces new elements such as the system's body measuring machine and the interpretation issues introduced in a virtual 3D environment. The various facets involved in the quality of delivery will be discussed later.

\subsubsection{Engagement}

Engagement as a method of increasing long-term compliance has been well researched in the discipline of exergaming and public health $[7,46,55,57,73]$. Drawing from video gaming and sports, exergaming has sought to attain an experience of flow where a balance between challenge and fun is combined with bodily exertion to create a sense of immersion $[15,57,66]$. Guidelines for exergame and system design have been developed but even commercial systems have not been able to demonstrate long-term commitment to exercise $[38,63]$. In clinical settings, the novelty of these games for physiotherapy has produced a surge in popularity as an alternative to the standard printout sheet.

Nintendo's Wii has become the most widely adopted interactive gaming system for rehabilitation, commonly referred to as 'Wiihabilitation'. Several publications have examined the success of Wii Sports in seniors' residence [1,6,42]. The enthusiasm was unexpected and has even escalated to residence-wide Wii bowling leagues and tournaments [42]. The Wii is effective in this situation because the kind of activity is not 
especially important, only that the seniors are engaged in the activity. The same activities would not necessarily be suitable as rehabilitation for patients with musculoskeletal injuries since needs are more deliberate and focused.

With clinical supervision, a gaming system can provide some patients with the necessary novelty and entertainment needed to keep patients motivated and interested in their recovery with limited risk. While these games are not designed for rehabilitation, the supervision compensates for the lack of feedback. To our knowledge, no studies have examined adherence or commitment in a home environment without lab controls or researcher oversight.

\subsubsection{Performance}

When recovery from an injury commences, the goal is to return to an active lifestyle as soon as possible. Most patients begin physiotherapy with good intentions and dedication to the recovery process. Strengthening exercises can be effectively performed with a physiotherapist present to provide customized instruction and feedback, however, while at home without supervision, misunderstood exercises performed incorrectly can undermine the effectiveness of the therapy, or even be harmful for the patient. According to Rainville et al. [60] the use of postural compensations during physiotherapy can promote pain and also reduce motor ability. While literature on patient compliance and sports commitment report self-efficacy as a major factor of engagement in an activity $[12,54,77,81]$, interactive gaming rehabilitation has not yet explored the importance of instruction delivery or user comprehension. Without a physiotherapist to provide custom instruction and clarification of queries, gaming systems for rehabilitation must pay 
special attention to methods of delivery, including appropriate modalities for the actions.

Particularly with yoga, some exercises require the patient to face away from the instructor or screen. If the instruction is unclear, craning the neck to verify positioning may make the patient susceptible to injury. In such cases, alternate modalities may be considered useful for safe interaction with gaming systems while ensuring patients feel confident and capable.

\subsection{Interactive Gaming For At-Home Physiotherapy}

In rehabilitation settings, anecdotal evidence and a series of case studies have provided encouraging results supporting the use of gaming. Patients have reported feeling more engaged by the gaming features and cared for by their physiotherapists in being provided up-to-date treatment methods [77]. However, the lack of larger-scale studies makes it difficult to determine the impact of the novelty of a gaming system as an alternative to repetitive exercise routine and its short-term and long-term benefits. Though some studies have developed devoted games or systems for academic analysis $[28,29,64]$, few to none have reached commercial success. Conversely, the potential limitation of the available commercial games and gaming systems, even though they encourage improvements in balance, strength, and fitness, is that they are not necessarily designed for rehabilitation in that they cannot be adapted for specific patient limitations and no correction of compensations is given $[26,28,33,71]$. 


\subsubsection{Body Measuring Machine}

Fitness instruction and yoga games are available for most interactive gaming systems today but are generally geared towards entertainment rather than accuracy. Games typically include a demonstration or training module preceding gameplay to teach users the action before they attempt to execute it themselves. Though this can mitigate the risk of injury, the quality of delivery can affect the comprehension of the action. By allowing for a wide range of acceptable motion these systems accommodate for varying audience ability and evidently, understanding, at the expense of motion measurement precision. As a result, the system accepts a wider range of error in the name of keeping the game fun and entertaining [41].

During execution, game feedback is limited to informing users of incorrect positioning rather than offering a way to correct. In some cases, because of the method of motion capture, no feedback is provided at all. For example, at least three poses in the Nintendo's Wii Balance Board Yoga game do not even use the balance board thus not collecting any motion information or having capability of providing feedback. In these cases, the system fails to utilize the interactivity that sets it apart from an average instructional video. Though this may be an appropriate design for entertainment it is not necessarily useful or well suited for rehabilitation or exercise training like Yoga, where precision and accuracy are important.

At the core of this issue is the body-measuring machine itself. The method and amount of body information collected determines the gaming system's feedback capability. Commercially successful systems often use one or more types of sensors to gather enough body information to create an engaging interaction or rapport with the 
user, however, this is not sufficient for providing constructive contextual feedback for the purpose of training or physiotherapy. For example, the Nintendo Wii Balance board uses pressure sensors that are used to measure the user's center of balance and weight. Though balance is an important component to many poses and exercises in the Wii Fit game, it does not speak to the accuracy of the other elements of the pose, which are not collected. Two problems can occur here: firstly, since success is determined by only one measure the weight distribution - pose accuracy, according to the system, may be achieved before the physical benefits from the other elements of the pose are attained, resulting in reduced effort by the user due to the system's affirmation. Secondly, inaccuracy of the other pose elements that are not measured can undermine the exercise itself and even lead to injury.

Confidence in one's ability is an important element to successfully complete a physiotherapy routine. However, due to limited body measurement collection, games like Wii Fit + Balance Board contribute to a patients' false sense of confidence. An inaccurate pose may produce encouraging words and praise from the system rather than constructive feedback, giving users an inflated sense of understanding and ability. Though the accuracy may not be designed for precision or rehabilitation, the Wii, and similar systems, do succeed in creating an engaging dialogue with the patient through feedback (though limited) and encouragement, which can contribute to regimen compliance. The system even verbalizes this compliance expectation when Wii's Yoga instructor points out that your wobbliness can be remedied by practicing everyday.

It is clear that some of the major discussions around musculoskeletal injuries in the realm of rehabilitation gaming have been mainly focused on encouraging long-term engagement in rehabilitation regimen $[9,24,49,74]$ or refinement of the motion capture 
precision of body measuring machine $[29,44,58,61,76]$. Although research in this area has made progress, a fundamental issue that seems to be overlooked is the quality of activity for musculoskeletal injury rehabilitation gaming.

Engagement and system accuracy of the body-measuring machine are both important aspects, however; literature that we have discussed previously on physiotherapy compliance has suggested that the effectiveness of information delivery is crucial to providing a successful and engaging at-home physiotherapy solution. An effective demonstration is often formed by the instructor's experience; compiling elements that they found useful when learning it themselves and also from observing others going through the process of learning. When gaming is introduced, that element of ad hoc feedback from personal experience is lost and a host of new issues regarding visio-spatial understanding and learning in a virtual 3D space surface. The aim of this study is to explore the variables that contribute to the quality of execution and user comprehension; this study will look at adapting for occlusion and lack of visibility, learning and orientation and providing feedback. We will examine each of these variables in detail next.

\subsection{Cognitive Interpretation Issues}

Comprehension of actions involves an array of cognitive abilities. Interactive gaming introduces new factors that can contribute to confusion. This thesis explores a selection of these factors, which include: 


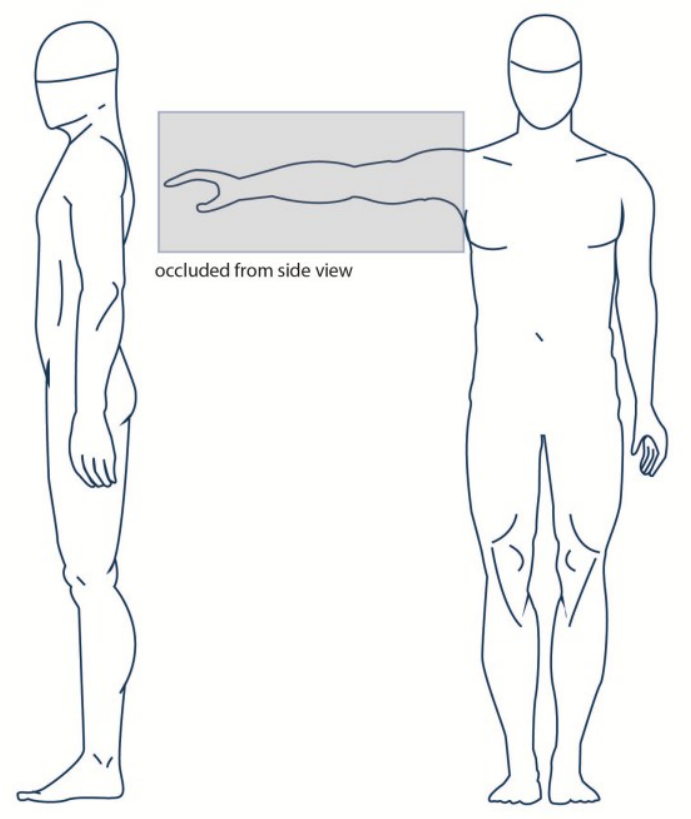

Figure 1 Side and Front View Demonstrating Occlusion

\subsubsection{Adapting For Occlusion}

For the purposes of this study, occlusion is defined as a visual obstruction that prevents the viewer from obtaining visual data required to complete an accurate mental image of the object viewed. Occlusion, which can be seen in figure 1, has implications in 2D and 3D instruction. During an in-person session the instructor has the freedom to adjust their own position to provide a viewpoint thought to be the best for the participant to understand all the target points of the pose. In yoga classes, instructors will often have mirrors behind them to assist in viewing hidden areas. The choice of viewpoint might be based on past personal difficulties or targeted to a participant's difficulties. Instructors may also address problematic areas with verbal descriptions, physically adjusting the patient or changing position to offer a new viewpoint that may reveal some occluded areas. 
While in-person instruction has addressed occlusion on an individualized basis, training videos and interactive games to a certain extent has not. Physiotherapists often provide patients with a sheet of printed exercises to perform at home. Like interactive pose games, these 2-dimensional flat images try to illustrate the angle that best shows the body movement, but occluded limbs or small body corrections may result in an incomplete mental image of the pose.

Designing a visual solution for providing multiple views or modalities without overwhelming the user and impairing their cognitive load can increase pose understanding and confidence and decrease the risk of injury. This thesis seeks to address this problem by demonstrating that current methods of pose demonstration, when not supervised by a physician, are commonly performed incorrectly and as a result, undermines the effectiveness of the activity.

\subsubsection{Sense of Self \& Orientation}

Understanding and awareness of oneself with relationship to others is an essential part of imitation and kinesthetic learning [56,78]. Where body language and communication between self and instructor address these challenges in-person, a virtual instructor, virtual space and virtual avatar to represent self, produce new learning challenges. Maintaining the users' sense of orientation of self and environment in comparison to their virtual avatar and the virtual environment is essential to learning through visual imitation and comparison to the instructor. Developing good design patterns to support learning and orientation requires a combination of psychology and aesthetic. Though it is important to determine whether a user learns better with a 
mirrored or frontal view, in some cases, it may even be difficult to determine which way the avatar is facing because of the avatar design.

Some elements that may be helpful to learning in an interactive pose game are unique to this medium. They include: the ability to focus only on the targeted areas for emphasis; graphical elements to show directionality; or panning around the avatar to create a continuous 3D visualization. Conversely, this may be challenging for those who become confused by the meaning of those elements, or lose overall orientation with a change in direction or without a full mental image.

While gaming systems have made it possible to offer a strategic angle change when necessary, the seamlessness of the demonstration can also impact a users sense of orientation. Daems et. al found that when participants were asked to select like-poses, being shown two images at rotation angles greater than 30 degrees in difference consecutively resulted in increased response times when compared to angles of lesser degree separations [16]. Kim et, al, approach this through demonstrating a pose as an interactive $2 \mathrm{D}$ avatar the user can walk around and examine [45]. Though this simulates a physiotherapist's demonstration more accurately, the self-explorative nature of demonstration lacks meaningful guidance. Designers need to be aware of learning patterns and delivery methods that might increase cognitive load, such as the one mentioned above, in order to develop helpful interactions that improve comprehension and as a result, user performance and confidence. 
A commonly mistaken solution to visual misinterpretation is to add additional graphical elements to assist with clarification, however, trying to overcompensate by adding more visual elements increases the cognitive load and can make understanding even more difficult. There are many variables to consider when designing for learning and orienting the user. The right ones can only be found with user input and testing. This thesis seeks to asses a selection of delivery methods from a quantitative and qualitative perspective to examine not only the actual performance value of a delivery but also the participant preference and confidence value.

\subsubsection{Feedback}

We define feedback as any visual, verbal or physical commentary of an individual's actions that lead them to achieve a target. Feedback is what sets in-person instruction apart from sheets of exercises, fitness videos and to an extent interactive game systems. While initial instruction usually demands the participant's full attention,
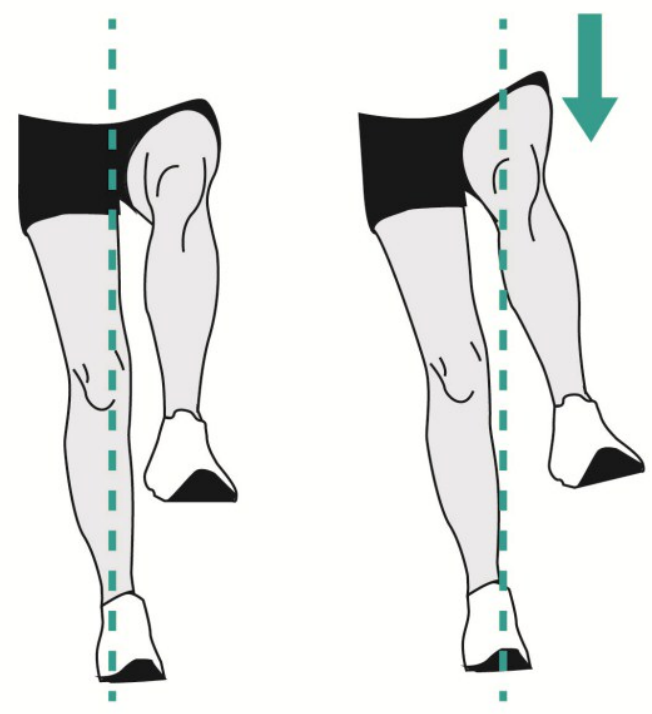

Figure 2 Correct Vs. Incorrect Knee Raises 
effective feedback has to consider the interpretation factors described above while considering the patient's context - in some cases, they may be mid-activity.

During in-person instruction, the instructor may demonstrate a pose that the participant may not understand with only one viewpoint but the advantage comes from the instructor's ability to provide supplementary visual feedback; by changing viewpoints to accommodate for occluded areas, provide verbal feedback; by describing the desired action, and even physical feedback; by gently moving the participant to the correct position. Providing constructive feedback and not just listing inaccuracies is something gaming instruction has yet to offer. For example, in figure 2, knowing the knee is in an incorrect position along with the information concerning the correction to bring the hip down, is more beneficial than simply knowing the knee is incorrect. In person, this feedback comes from the experience that the solution is co-related to other areas of the body and not only the point of error. Similarly, in interactive gaming system's, feedback can be divided into three requirements; the gathering of body information by the systems sensors, the system's interpretation of the information with appropriate response, and finally, how the response is delivered to the user. While an ideal interactive gaming system design would be intelligently aware of the user's activity from body measurement and would adapt modally to suit the user's capability. In reality, as discussed in section 2.2.1 on Body-Measuring Machines, depending on the system, constructive feedback may be very difficult or impossible to provide if detailed body information is not collected.

While a significant amount of literature in exergaming and gaming for physiotherapy has focused on developing more suitable methods of collecting and 
interpreting body information by the system, exploration has been limited when it comes to delivering meaningful (contextually but also in an appropriate modality) and constructive criticism. 


\section{Test 1 Demonstration Delivery}

During the demonstration delivery study, participants were tested on their understanding of yoga poses delivered by a $2 \mathrm{D}$ animated instructor meant to replicate the visual style of a video game avatar. The intent is to determine a patient's confidence and perceived accuracy of a pose in comparison to actual pose accuracy while manipulating the visual delivery and verbal delivery.

\subsection{Task}

The study was approved by the Carleton University Research Ethics Board to conduct research involving humans. Participants were described the purpose of the study before beginning and signed a consent form confirming they understood. They were then verbally asked a number of introductory experience and demographic questions which were recorded on paper (pre-test questions can be found in appendix B.1). Each participant used a $15^{\prime}$ laptop to watch 2 sets of yoga pose demonstrations with five in each set, for a total of 10 different yoga poses. Figures 3 and 4 illustrate the respective poses used for each section.

Demonstration videos were created for the study using Maya animation software to the specifications of the visual delivery required for the study. Poses, verbal description and pose elements, were derived from Nintendo Wii's Yoga game poses unless a particular pose was not available. In these cases a standard Bikram Yoga pose was used. 
Set 1 Poses Visual Delivery

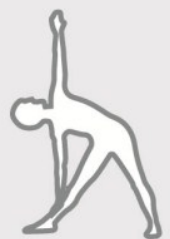

Triangle

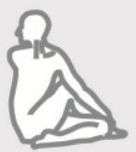

Half Spine Twist

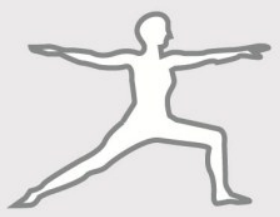

Warrior II

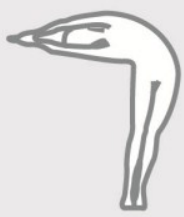

Halfmoon

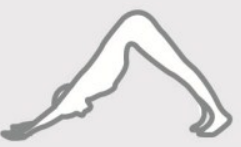

Downward Dog

Figure 3 Demonstration Delivery, Set 1 Poses

Set 2 Poses Verbal vs. Non Verbal

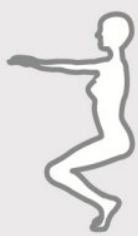

Chair

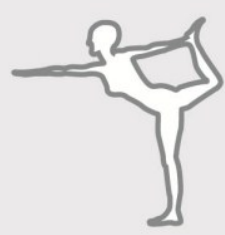

Dancer

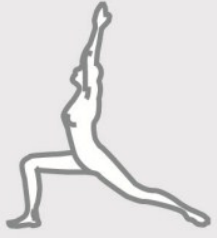

Warrior I

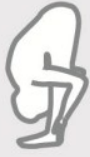

Forward Fold

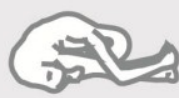

Seated Head to Knee

Figure 4 Demonstration Delivery, Set 2 Poses

\section{Set 1 Conditions Visual Delivery}
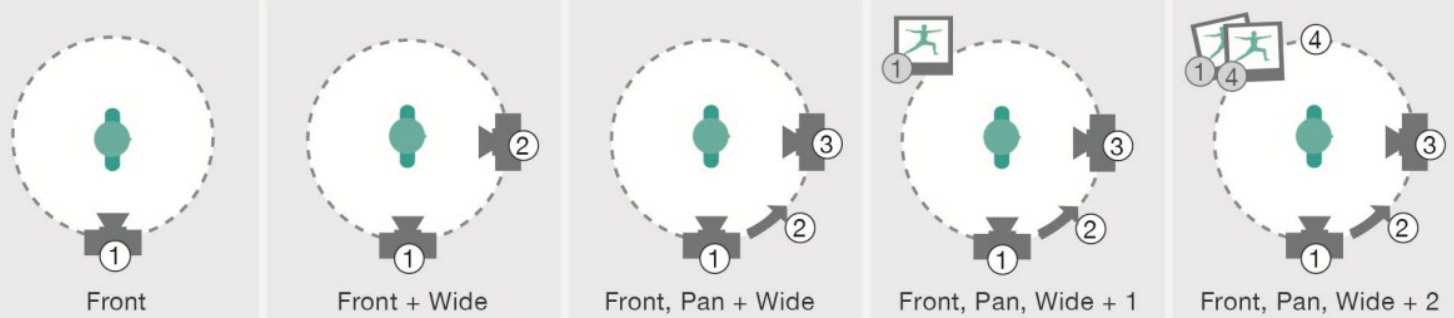

Front, Pan + Wide

Front, Pan, Wide +1

Front, Pan, Wide +2

Figure 5 Demonstration Delivery, Set 1 Conditions

Set 1 
The first set of the demonstration delivery test focused on the visual delivery. Participants all saw the same poses in a random order matched with a random condition. All Participants saw all conditions. The conditions, illustrated in figure 5, include:

1. Front: The most occluded view type.

2. Front + Wide: The second begins with a frontal viewpoint then switches to the viewpoint that captures the widest visual area of the instructor - this view type offers an additional angle from the front angle to improve visualization of occluded areas.

3. Front, Pan + Wide: The third begins with the frontal view, but will pan around the instructor to 40 degrees while they are performing the exercise. The viewpoint then switches to one that captures the widest visual area of the instructor - This view type offers a smoother transition between the same angles in the last view type.

4. Front, Pan + Wide $x 1$ : The fourth pose will provide the pose in the same manner as the third but will offer an additional supporting viewpoint while the widest viewpoint is displayed - This view type reveals three angles throughout the demonstration, making occluded areas minimal.

5. Front, Pan +Wide $x 2$ : The fifth will be similar to the fourth but offer two supporting viewpoints while displaying the widest viewpoint - this view type offers two additional images for a total of 4 angles throughout the demonstration making it the least likely to offer occluded areas. The amount of information displayed in this demonstration is what we anticipate will be a visual/cognitive peak. 


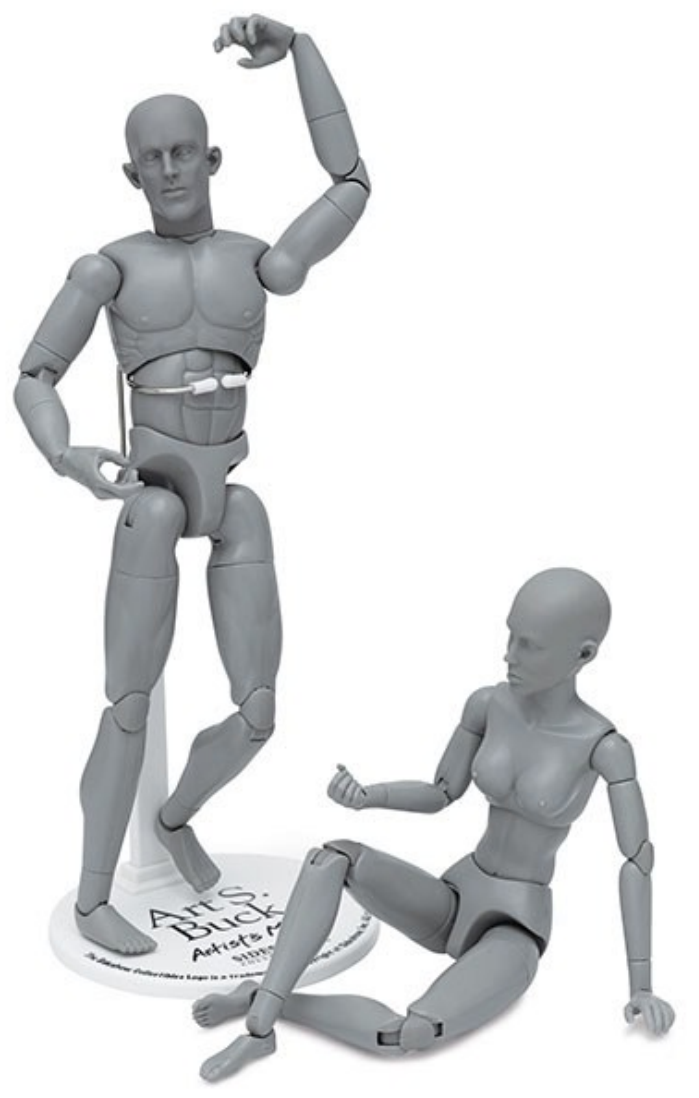

Figure 6 Flexible Manikins

Participants were able to watch the demonstration twice and were then asked to replicate the final pose using a flexible manikin ${ }^{1}$, seen in figure 6 . Both male and female manikins were available to participants. The final image of the demonstration was left on screen while the participant posed their manikin. To compensate for the lack of range of motion of the manikins and also to obtain a clearer idea of participant understanding of physical subtleties of the pose, participants were asked to verbally describe the elements of their posed manikin. With 6 years of regular Bikram Yoga practice, the researcher scored pose accuracy after each pose, based on a set of elements in the demonstrations. The score criteria can be found in Appendix D.1. After each pose the researcher asked

\footnotetext{
${ }^{1}$ Art S. Buck Sideshow Collectible ${ }^{\circledR}$ Anatomical Models image collected from http://www.dickblick.com/products/arts-buck-anatomical-models/
} 
participants to verbally score their confidence in understanding, clarity of the demonstration and perceived pose accuracy on a 5-point Likert scale. Questions can be found in the study script in Appendix A.1. Comments and criticism on experience were transcribed by the researcher using a laptop.

Set 2

The second set of demonstration videos in the demonstration delivery test focused on verbal + visual vs. visual only delivery. Like the last set, participants were shown a visual demonstration in a consistent visual delivery on a 15 ' laptop. In this section, however, the poses were provided in a random order with a random condition - with or without verbal description. Again, participants were able to watch the demonstrations twice and had to then pose and describe the manikin. They were able to see the final frame of the video while posing the manikin, just as in the first set. After each pose, they were scored by the researcher and asked the same questions as the previous set. They were not informed of their pose scores. Upon completing all 10 poses, participants completed a short exit survey (found in Appendix C.1) about their preferences of visual and verbal delivery.

\subsection{Design}

\section{Set 1}

In the visual demonstration section of our study we used several $5 \times 1$ repeated-measures within-subject design. Our factors were: Visual demonstration deliveries (Front, Front Wide, Front Pan Wide, Front Pan Wide x1, Front Pan Wide x2) and individually assessed scoring measures: (pose accuracy scores, confidence, and perceived pose accuracy, 
demonstration quality). Each participant performed all 5 poses with the manikin (Warrior II, Half moon, Chair, Spine Twist, Dancer) that were randomly combined with one of the visual conditions. Each participant performed each condition. Combinations were randomly ordered for each participant. The experimental sessions lasted about 45 minutes.

Set 2

The second section of the demonstration delivery study focused on verbal delivery. Here we performed a paired-sample T-Test of verbal and non-verbal conditions, on each of the scoring measures to determine if there was an effect on scored performance, confidence and perceived pose accuracy.

\subsubsection{User Feedback}

After each pose participants were asked to rate their confidence in understanding of the pose, the quality of demonstration and their perceived pose accuracy. The questions were structured using a 5 -point Likert scale $(1=$ Strongly Disagree to $5=$ Strongly Agree). They were also given the opportunity to explain their rating. These responses were transcribed and coded using grounded theory [32]. In addition, participants were asked to complete an exit questionnaire where they had to identify the most helpful visual delivery followed by the least. They were then asked to rate the helpfulness of each of the visual deliveries using a 5-point Likert scale $(1=$ not helpful at all to $5=$ very helpful). 


\subsubsection{Participants}

A total of 40 participants volunteered to complete the experiment. They were recruited through posters around Carleton University campus and also a posting in The Graduate, Carleton's online newsletter. 20 participated in Section 1 (set 1 and 2), the demonstration delivery portion, with equal amounts of males and females (10 male, 10 female). The mean age of participants was 30 years old. On average, participants reported to be in above average physical health. Participants were asked to report their exposure and frequency to video games and also instructional fitness. A mode of 11 participants said they played video or mobile games 1 to 2 hours a week. 18 of 20 participants said they had taken part in an instructional yoga or fitness class at some point, however, as seen in table 1, a majority of those participants had only been to a few.

\begin{tabular}{lccccc} 
& Never & $\begin{array}{c}\text { I used to } \\
\text { but don't } \\
\text { anymore }\end{array}$ & $\begin{array}{c}\text { I've been a } \\
\text { couple times }\end{array}$ & $\begin{array}{c}1-2 \\
\text { times/week }\end{array}$ & 3+ times/week \\
\hline Males & 2 & 3 & 3 & 2 & 0 \\
Females & 1 & 2 & 3 & 3 & 1 \\
\hline ALL & 3 & 5 & 6 & 5 & 1
\end{tabular}

Table 1 Demonstration - Frequency of instructional class attendance of males and females

\begin{tabular}{lcccc} 
& None & Yoga & Fitness & Both \\
\hline Males & 2 & 2 & 3 & 3 \\
Females & 0 & 0 & 1 & 8 \\
\hline ALL & 2 & 2 & 4 & 11
\end{tabular}

Table 2 Demonstration - Distribution of instructional class type for males and females. 
A quarter of participants said they used to attend classes but do not anymore, while another quarter said they attend an instructional class at least once or twice a week. Males and females had a similar amount of exposure to instructional fitness class environments though women generally had slightly more yoga experience. Table 2 further displays a breakdown of experience into yoga vs. fitness.

\subsection{Hypotheses}

\section{Set 1}

We hypothesize that participants' comprehension score will be best when offered a single, short but continuous, multi-view demonstration that will ensure any obstructed or occluded pose elements are clarified - Front, Pan + Wide (H1). Suggesting that too many additional images will be visually overwhelming to participants (H2) and that they will score lowest when presented with only a front view of the pose (H3).

We predict confidence ratings will follow pose accuracy scores. We expect participants will feel most confident in their understanding of poses offering a single, short but continuous, multi-view demonstration (H4).Conversely, we predict participants will feel least confident when provided only a single view of a pose (H5).

\section{Set 2}

Although visual delivery is important it is often not enough on its own to gain a comprehensive understanding of an exercise, particularly in situations where the user must avert their gaze to complete the pose correctly. For this reason we felt it was appropriate to evaluate the impact of a supporting modality on performance. The effect of 
visual pose demonstrations with or without verbal description to accompany will also be evaluated. Under these conditions we anticipate that participants would score higher and also rate confidence higher if they were provided with verbal description of the pose in addition to visual $(\mathrm{H} 6, \mathrm{H} 7)$. Conversely, they would score lower when only provided visual demonstration (no verbal) (H8).

\subsection{Results}

Set 1

We began by looking for an effect of visual demonstration on pose accuracy. In this case, we predicted that the single, short but continuous, multi-view demonstration or Front, Pan + Wide would illicit improved pose accuracy. Figure 7 presents the mean pose accuracy scores and standard deviation across visual demonstration types. A repeated measure ANOVA did not reveal a significant relationship between pose score and visual demonstration conditions, $F(5,95)=.842, p>.05$. partial $\eta 2=.042$.

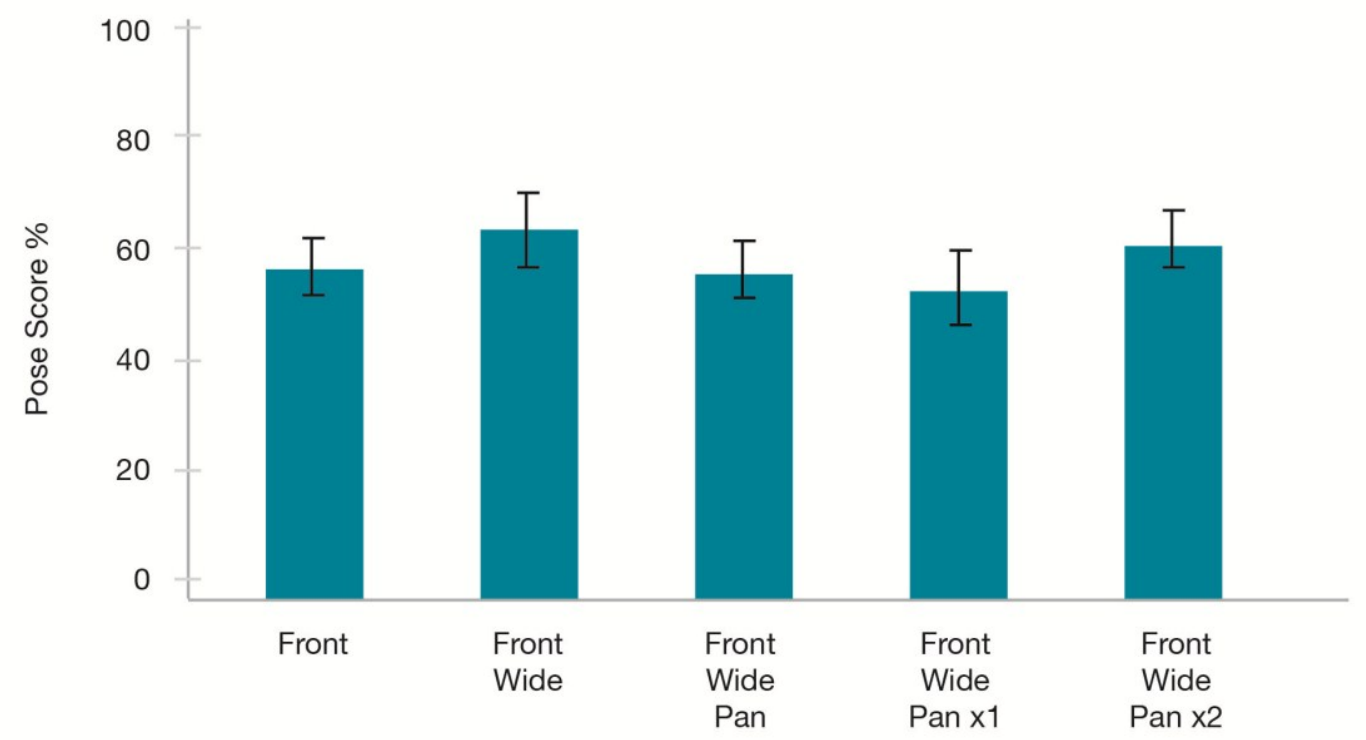

Figure 7 Mean Pose Scores (\%) for Visual Delivery Conditions 


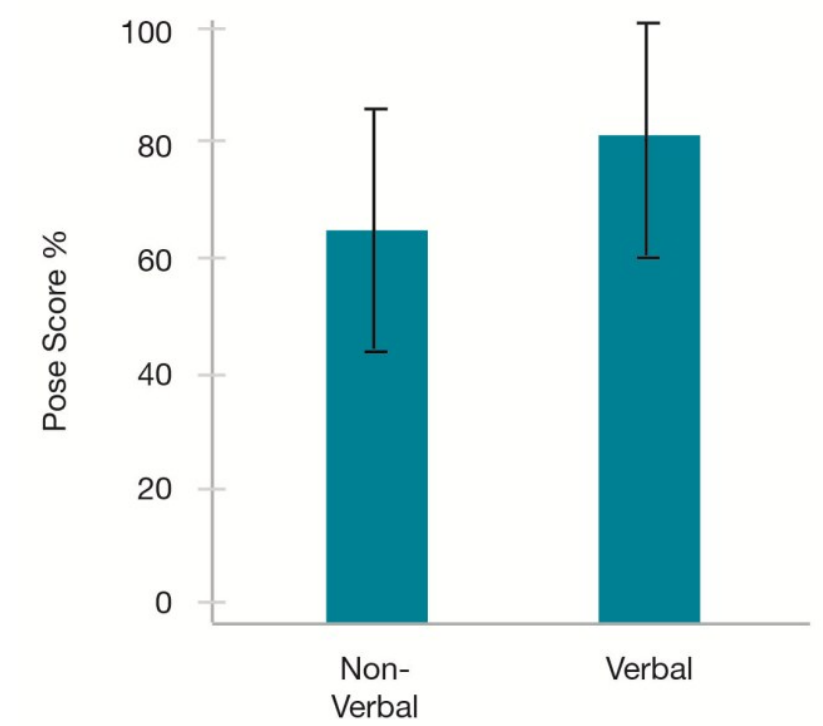

Figure 8 Mean Pose Scores (\%) for Verbal Delivery Conditions

Set 2

A paired-samples T-Test was performed on pose scores for verbal and non-verbal conditions. We discover that there is, in fact, a statistically significant effect of verbal conditions on pose score, $t(19)=2.132, p=.046, d=0.48$, where the verbal condition improved performance by $11 \%$. It is clear from figure 8 that participants performed better with verbal description than without.

\subsubsection{User Feedback}

Descriptives of responses to the 5-point Likert scale questions in the visual delivery section asked after each pose regarding confidence in understanding, quality of demonstration and perceived pose accuracy can be see in table 3 . 


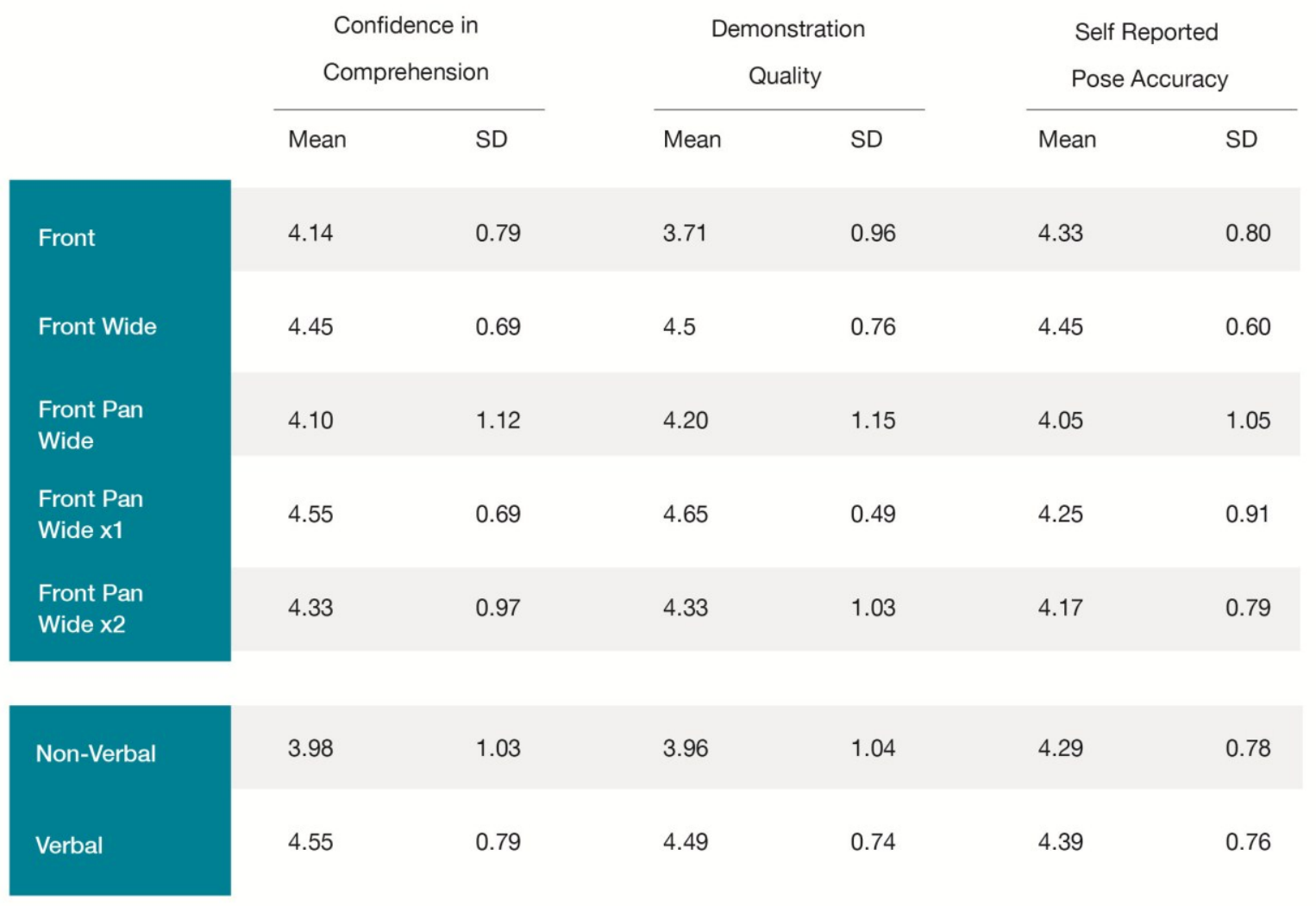

Table 3 Post-Pose Likert responses for Demonstration Delivery

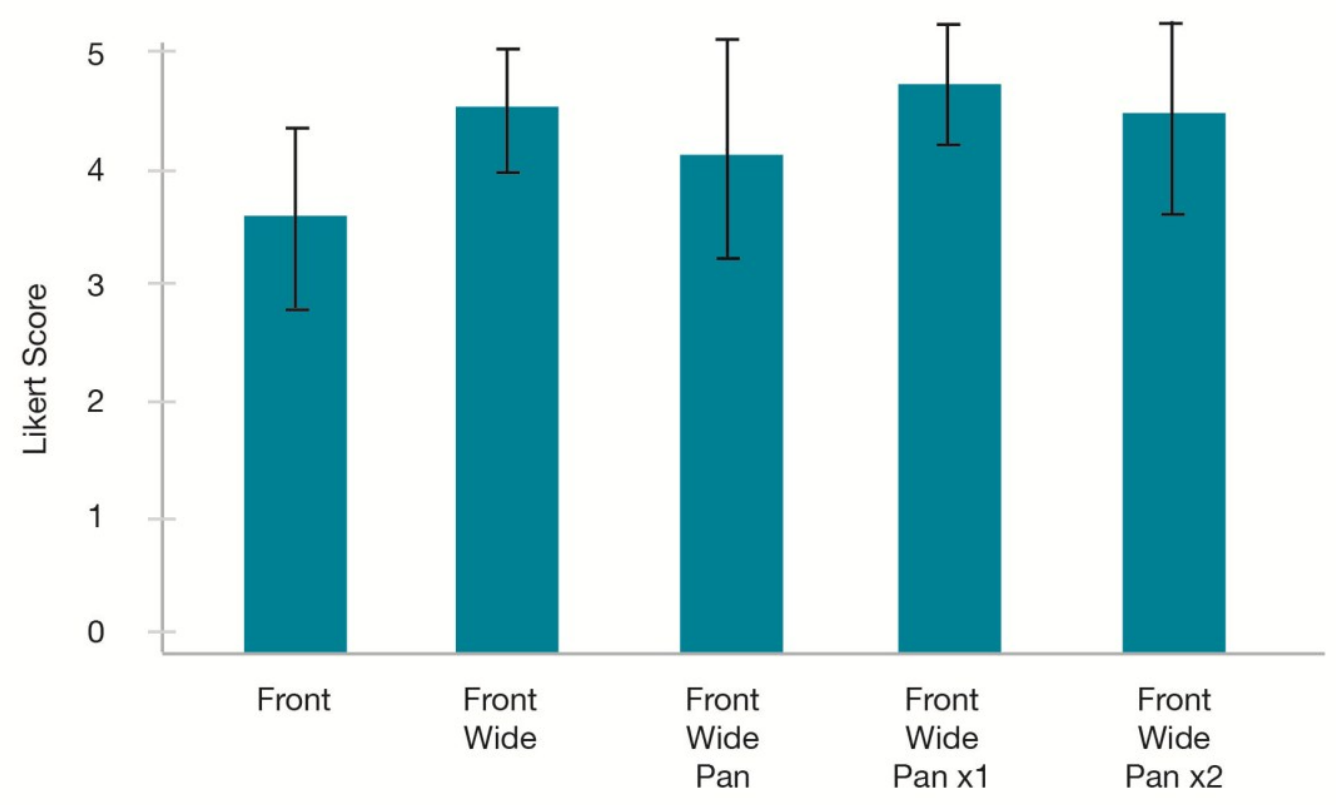

Figure 9 Mean Likert scores of quality of demonstration for Visual Delivery Conditions 
A repeated measures ANOVA analysis did not reveal a significant relationship between confidence and visual demonstration conditions, $F(5,95)=.842, p>.05$, or perceived pose accuracy and visual demonstration, $F(4,76)=1.121, p>.05$. However, when examining the mean Likert scores in figure 9 of participant-reported demonstration quality, we can see that the Front view type was scored much lower than the remaining types. In support of this observation, analysis using the Greenhouse-Geisser correction revealed a significant effect of demonstration quality preference on visual demonstration, $(F(2.853,54.205)=3.361, p<.027$. partial $\eta 2=.150$.). Post-hoc analysis with a Boneferroni adjustment further clarify the significant differences between the Front view and the other view types, suggesting participants found the Front view of lesser quality.

We then examined the responses to post-pose questions on confidence in understanding, quality of demonstration and perceived pose accuracy for the verbal section (Table 3). A paired-samples T-Test was performed on each of the Likert questions with verbal vs. non-verbal conditions. We discover that there is a statistically significant effect of verbal conditions on confidence, $t(19)=3.356, p=.003, d=0.75$ and also quality of demonstration, $t(19)=2.396, p=.027, d=0.54$. This suggests that participants, not only prefer being provided verbal description to support a demonstration, they are more confident in their understanding when provided with it. We did not find any significant effect of verbal conditions on perceived performance.

Following analysis of collected quantitative data, we began exploring individual participant responses. These responses were transcribed and coded using a Grounded Theory method [32]. Categorical responses can be found on page 44, Table 4 (Set 1) and 45, Table 5 (Set 2). 


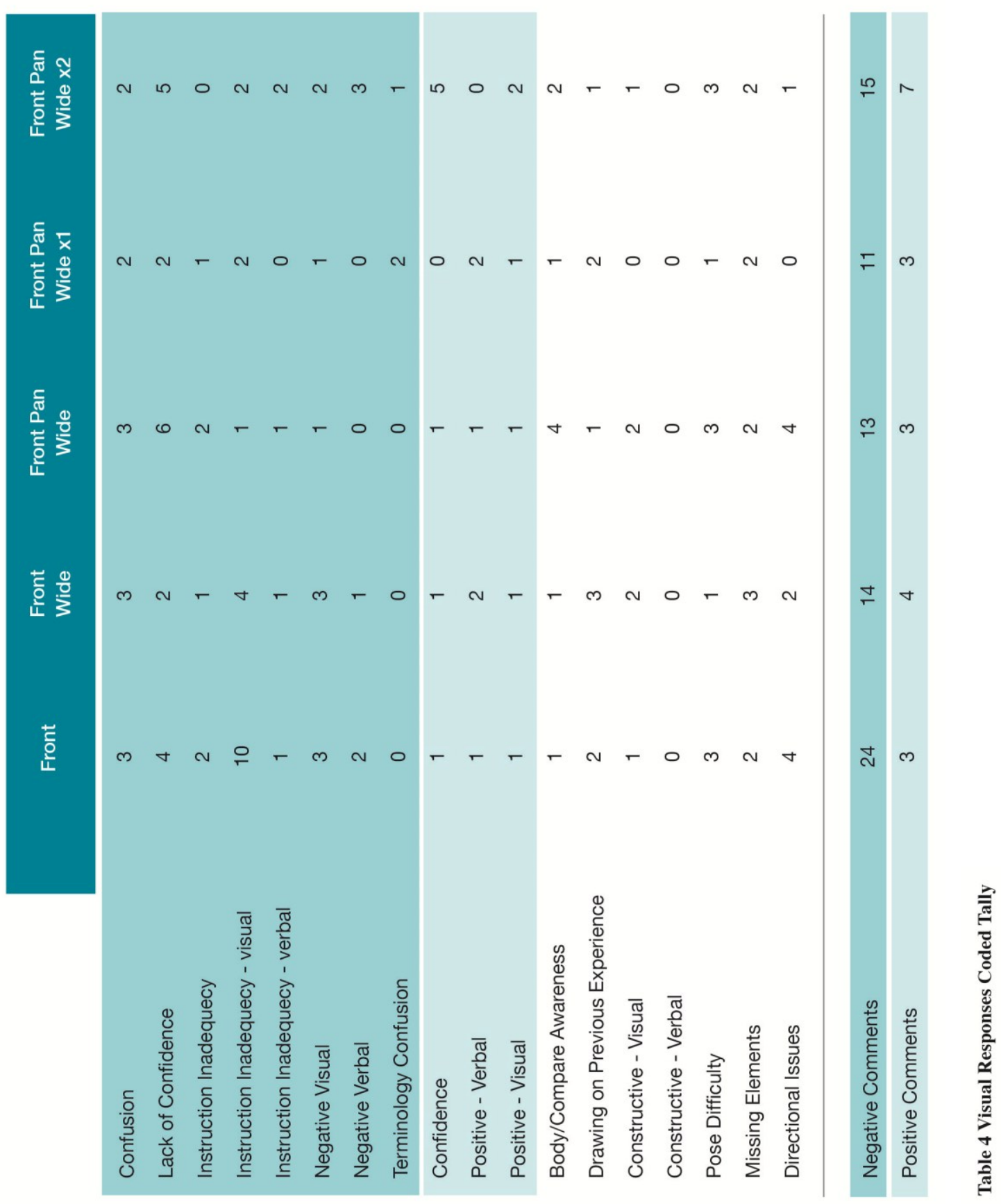




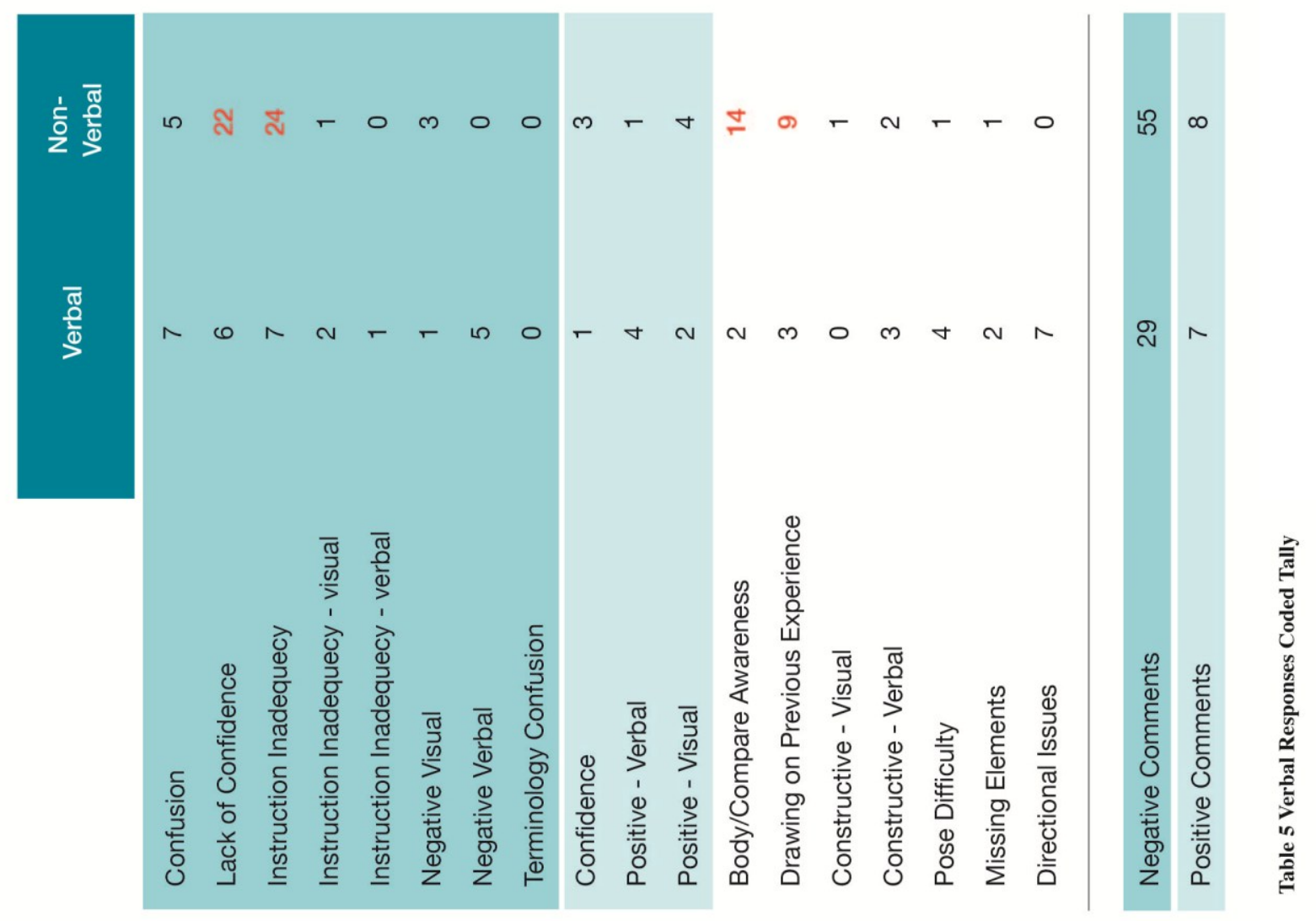




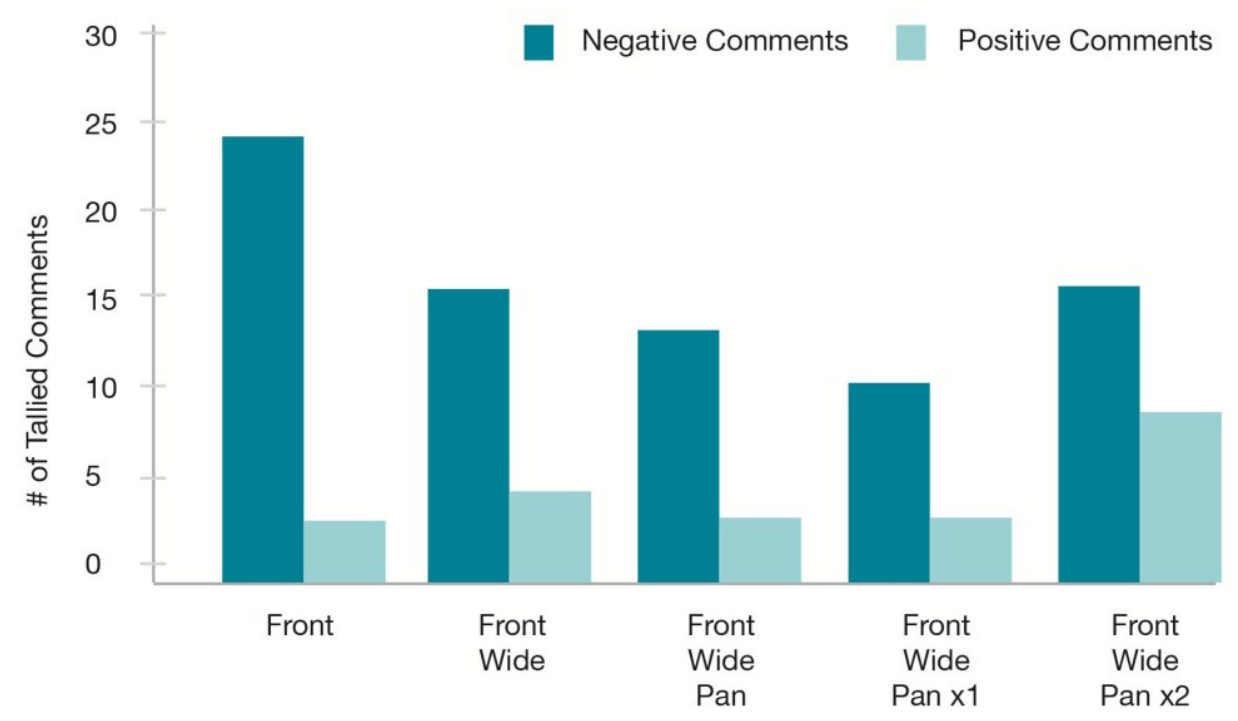

Figure 10 Grouped Negative \& Positive Comments for Visual Deliveries

\begin{tabular}{lcc} 
Category & Verbal & Non-verbal \\
\hline \hline Lack of confidence & 6 & 22 \\
Instruction inadequacy & 7 & 24
\end{tabular}

Table 6 Categorical tally of comments on verbal delivery

Categories were combined to form groups of 'negative comments' and 'positive comments'. From figure 10 we can see that the front view produced the highest number of negative comments and, in fact, received significantly more comments relating to the visual inadequacy of the demonstration, specifically. Panning view with 1 additional angle received the least number of negative comments. Conversely, the panning view with 2 additional angles received the most amount of positive comments further supporting the notion that a panning view with additional 
angles is the most preferred type of visual delivery for 3D virtual demonstration. Table 6 summarizes the contrast in comment sums for lack of confidence and instruction inadequacy for verbal and non-verbal demonstration, which further supports the statistical analysis.

At the end of the study, participants were asked to rate the most helpful type of visual feedback and the least helpful. The panning view with 1 additional angle was found to be the most helpful with a mean rating of $3.55, \mathrm{STD}=1.05$. The front view was the least helpful with a mean rating of $1.35, \mathrm{STD}=0.99$. Participants deemed the wide view angle - the most visible surface area of the instructor - more important than the other visual elements. A repeated measure ANOVA analysis revealed a statistically significant effect of demonstration deliveries, $F(4,64)=5.325, p<.001$. partial $\eta^{2}=$ .250. Post-hoc analysis with a Boneferroni adjustment revealed that participants significantly prefered the wide view compared to the front pan wide $x 2$ (1.059 (95\% CI..156 to 1.961), $p=.015$ ). While additional views were, on average, less helpful than other visual elements. The exit questionnaire also further supported the trend that verbal delivery was very important to participants, receiving a mean rating of 4.65 on a Likert scale of helpfulness, STD $=0.67$.

\subsection{Discussion}

\section{Set 1}

Though we predicted a single, short but continuous, multi-view (pan) demonstration Front, Pan + Wide - would perform best, statistical analysis found no significant difference in pose accuracy across each of the visual demonstration deliveries $(\mathrm{H} 1, \mathrm{H} 2$, 
H3). While not significant, the increase in mean pose accuracy from 1 additional image to 2 may indicate that we have yet to reach a peak of cognitive load. For the purposes of the experiment, we felt that two additional angles to the main demonstration would suffice as an example of excessive information but performance did not decrease (H2). In that case, further research exploring greater amounts of visual information to determine where the peak of cognitive load lies.

From questionnaire feedback and verbal account we can tell that participants were overall most positive about the panning view with 1 additional angle but most confident with 2 additional angles. While this is not what we expected (H4), in the absence of feedback, the additional views may have given participants a sense of confidence by offering additional points of reference to self-check their own pose accuracy.

The front view demonstration type provided a highly occluded perspective of the pose. While a high number of participants noted the poor visual quality of this feedback type this was not reflected in the pose accuracy scores. The front view was not found to have a significant effect on pose accuracy or confidence. Additionally, participants reported almost double the amount of negative commentary on the front view than any other visual delivery, specifically, more than double the amount of comments regarding the visual inadequacy. This seems to support the notion that, in the absence of feedback, the amount of reference points to self-check one's pose accuracy can impact the confidence and self-efficacy of a user. Future research in interactive gaming demonstration might assess the impact of varying amounts of visual information, including more than 2 additional angles, on performance and cognitive load and what that means for user confidence. 
We found that some of our hypotheses were not confirmed. Participant scores were not found to have a significant difference between self-reported confidence in understanding (H5) or with perceived performance ratings (H4).

Set 2

Verbal description to supplement visual demonstration was found to have a significant effect on pose accuracy and also confidence in understanding compared to visual demonstration with no verbal description (H6, H7, H8). From verbal account, participants noted that they were better able to visualize how to get into the pose, where they should feel a stretch and how to correctly position themselves better with verbal description than without. Without description participants said they were unsure about where they should feel the stretch and overall did not feel confident in their understanding. This was also reflected in high scoring of verbal delivery in the exit questionnaires.

Literature on cognitive understanding of action has explored whether a pose is understood as many different angles that are related to one pose or one pose that we mentally rotate $[16,62]$. Daems et al. found that when participants were asked to match like poses, response time increased when an angle changed greater than 30 degrees [16]. This research motivated us to explore this visual interpretation by setting our pan to 30 degrees and our angle jump to 90 degrees. In trying to keep the thesis scope focused we did collect verbal account of participant feelings and commentary on these changes, but we did not measure response time. We expected that pose accuracy would not be affected but predicted we would see preference for the smoother more natural panning view to the 
rigid angle jump. This was supported by verbal accounts. Some noted that the angle jump felt jerky. The exit questionnaire Likert scores also revealed that poses with a pan were much more preferred to those without. Future research might explore the impact panning vs. angle jumping and the max and min angle jump on response time, pose accuracy and confidence. 


\section{Test 2 - Feedback}

The intent of this experiment is to find an optimal method of delivery that facilitates full exercise comprehension in an effort to decrease potential injury in an athome physiotherapy environment. Many gaming systems offer poor to no feedback once they have demonstrated an activity, at best, informing the user of incorrect placement but rarely telling the user how to correct. Without feedback, this type of delivery is comparable to an exercise video. People may not even realize they are performing the activity incorrectly putting them at risk for injury. These concerns will be assessed in the following experiment.

\subsection{Task}

The study was approved by the Carleton University Research Ethics Board to conduct research involving humans. During the Feedback study, participants were tested on comprehension of yoga poses, before and after feedback, delivered by Nintendo's Wii Fit Yoga game and Balance Board [59]. The Wii was used as a demonstration platform to support the assessment of the Wii graphical + visual feedback against the other feedback types in an effort to determine current commercial system effectiveness.

The intent of this experiment is to find an optimal method of feedback delivery that can prevent injury during exercise in an at-home physiotherapy environment. Different types of feedback were assessed, including visual, verbal, haptic and combinations of feedback. 


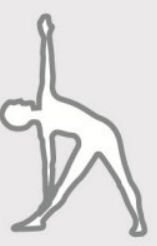

Triangle

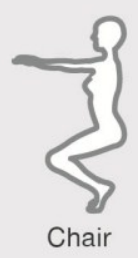

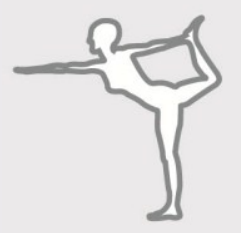

Dancer

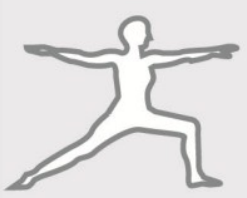

Warrior II

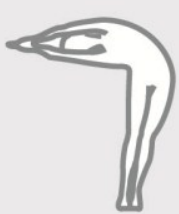

Halfmoon

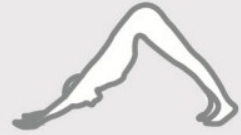

Downward Dog

Figure 11 Feedback Poses

\section{Feedback Conditions}

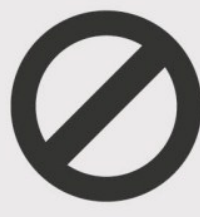

No Feedback

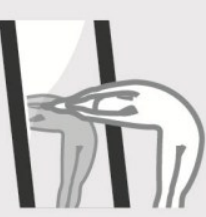

Mirror Feedback

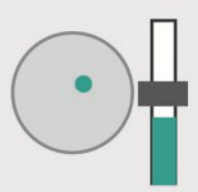

Wii Graphic + Verbal Feedback

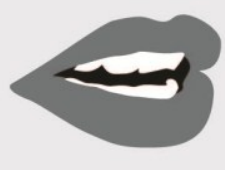

Custom Verbal

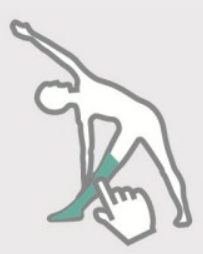

Haptic

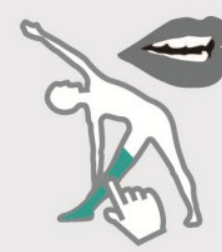

Custom verbal + haptic

Figure 12 Feedback Conditions

Like the last study, participants were described the purpose of the study before beginning and signed a consent form confirming they understood. Before arriving, participants were asked to wear comfortable exercise-type clothing for the study to reduce pose difficulty from clothing. We have tried to mitigate the 'white coat effect' by having only one researcher/scorer in the room with the participant. The researcher dressed in comfortable yoga clothing and tried to make participants feel as comfortable as possible before starting. The room used was spacious and participants were encouraged to lightly stretch before beginning.

To start, they were then verbally asked a number of introductory experience and demographic questions by the researcher which were then recorded on paper (pre-test questions can be found in Appendix B). To complete the experiment, participants 
interacted with Nintendo's Wii Fit Yoga fitness game and balance board. Six yoga poses, seen in figure 11, were selected from the game and were delivered as a large projection in demonstration mode (rather than play mode) in a random order. Each participant was exposed to each condition. The researcher used the Wiimote to select the appropriate pose. The participants watched the demonstration once and executed the pose during the second demonstration along with the 2D instructor. During execution, participants received a randomly chosen feedback condition to help correct any pose errors. The feedback conditions, which can be seen in figure 12, included:

- No feedback: As a baseline.

- Mirror Feedback: To determine the effectiveness and value of a participant's own mirrored body as comparative feedback. Yoga studios and physiotherapy clinics often use mirrors to observe positioning and posture. Similarly, some gaming systems use on-screen avatars to offer users a method of visually comparing their position to the virtual instructor. The Mirror used was a long rectangular full body mirror measuring $59^{\prime}$ X $15^{\prime}$. It was placed in front of the participant careful to not obstruct the demonstration.
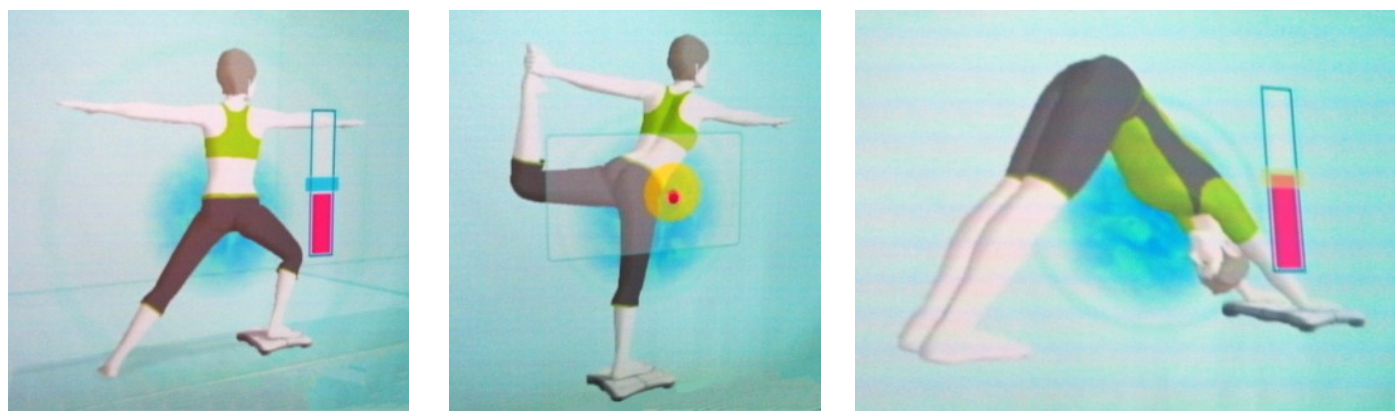

Figure 13 Wii Fit Yoga Feedback Based on Balance Board Measurement 
- Wii Yoga's built-in graphic and verbal feedback: Mechanism measured by the balance board. To evaluate the effect of the combination of graphical and verbal feedback driven by limited body measurement information on participant performance and confidence. The type of feedback can be seen in figure $13^{2}$.

- Custom verbal feedback: Delivered by the researcher that is detail-focused.

Simulates the effect of a richer body measuring system and evaluate the importance of more comprehensive body measurement information on performance and confidence.

- Haptic feedback: Simulated by tapping participants on an incorrectly positioned area. This condition is meant to imitate the assistive touch of a physiotherapist putting a patient into the correct position and evaluate the effect of an alternate supporting modality, such as touch, on performance in a gaming environment.

- Haptic and verbal feedback: Combining the last two conditions to simulate the modalities utilized at an in-person session.

Feedback was based on the scored elements directly from the demonstration and was provided for the duration of the onscreen instruction - about 1 minute. Participants received feedback only when position was incorrect.

With 6 years of regular yoga practice, Pose accuracy was scored by the researcher, based on a set of elements in the demonstrations (Appendix D.2). Participants then verbally scored their confidence in pose understanding before feedback and after

\footnotetext{
${ }^{2}$ Condition 3 requires the participants to use play mode rather than practice mode for the execution playback, once they have seen the initial demonstration.
} 
receiving feedback as well as perceived pose accuracy on a 5-point Likert scale. The questions can be found in the study script in Appendix A.2. Comments and criticism on experience were also transcribed by the researcher using a laptop. Participants were not informed of their pose accuracy scores. Upon completing all 6 poses, participants completed a short exit survey about their preferences of feedback type (Appendix C.2). Participants were reminded that if a condition wasn't clear that they could be shown an example to assist in answering the questions.

\subsection{Design}

We used a 6x1 repeated-measures within-subject design. Our factors included: feedback type (baseline-no feedback, mirror feedback, Wii graphic + verbal feedback, custom verbal feedback, custom haptic feedback and custom verbal + haptic feedback) and individually assessed scoring measures: (pose accuracy scores, confidence, and perceived pose accuracy). Each participant performed all six pose possibilities (Triangle, Half moon, Chair, Warrior I, Downward Dog, Dancer) randomly combined with each of the feedback types to complete 6 combinations. Conditions were randomly ordered for each participant. The experimental sessions lasted about 40 minutes.

\subsubsection{User Feedback}

After each pose, participants were asked to rate their confidence in understanding of the pose before they received feedback, after they received feedback and their perceived pose accuracy. The questions were structured using a 5-point Likert scale $(1=$ Strongly Disagree to $5=$ Strongly Agree). They were also given the opportunity to 
explain their rating. These responses were transcribed and then coded using grounded theory [32]. In addition, participants were asked complete an exit questionnaire where they had to identify the most helpful visual delivery followed by the least. They were then asked to rate the helpfulness of each type of feedback using a 5-point Likert scale (1 $=$ not helpful at all to $5=$ very helpful).

\subsubsection{Participants}

Twenty participants were recruited to complete the second study who had not participated in Section 1. There were almost equal amounts of males and females (11 male, 9 female). The mean age of participants was 29 years old. On average, participants reported to be in above average physical health. Participants were asked to report their exposure and frequency to video games and also instructional fitness. A mode of 9 participants said they never play video or mobile games. Three quarters of the 20 participants said they had taken part in an instructional yoga or fitness class at some point, but as you can see in Table 7, a majority (6) of those participants had only been to a few. A quarter of participants said they had never attended an instructional class, while 4 used to attend classes but don't anymore and 1 said they attended once or twice a week. Males and females had a similar amount of exposure to instructional fitness class environments but women typically had more yoga experience than men. Table 8 distributes experience into yoga vs. fitness. 


\begin{tabular}{lcccc} 
& None & Yoga & Fitness & Both \\
\cline { 2 - 5 } males & 4 & 1 & 4 & 2 \\
females & 1 & 2 & 1 & 5 \\
\hline ALL & 5 & 3 & 5 & 7
\end{tabular}

Table 8. Feedback - Distribution of instructional class type for males and females

\subsection{Hypotheses}

Having looked at the effect of demonstration delivery on initial comprehension, the final aspect we examine is the effect of feedback on performance and confidence. As we have discussed, feedback is an essential part of fully grasping a pose or activity.

In this study we are interested in physical performance, in particular, the physical adjustments and understanding of the criticism, if any exists. We are also interested in participant feelings and preference for each type of feedback. Using these modes of feedback, we anticipate that participants would perform best (H9) and feel most confident in their pose understanding (H10) when provided with customized verbal and haptic feedback that replicated feedback delivery offered by a physiotherapist. We also predict that because the Wii offers feedback only for a particular aspect of the pose, balance, and not necessarily the primary objective of the pose, Wii feedback with balance board would improve confidence but not performance score (H11).

\begin{tabular}{lccccc} 
& \multicolumn{2}{c}{$\begin{array}{c}\text { I used to but } \\
\text { don't } \\
\text { anymore }\end{array}$} & $\begin{array}{c}\text { I've been a } \\
\text { couple } \\
\text { times }\end{array}$ & $\begin{array}{c}1-2 \\
\text { times/week }\end{array}$ & 3+ times/week \\
\cline { 2 - 6 } Males & Never & 1 & 4 & 1 & 1 \\
\hline ALL & 1 & 3 & 2 & 0 & 3 \\
\hline
\end{tabular}

Table 7. Feedback - Frequency of instructional class attendance of males and females 


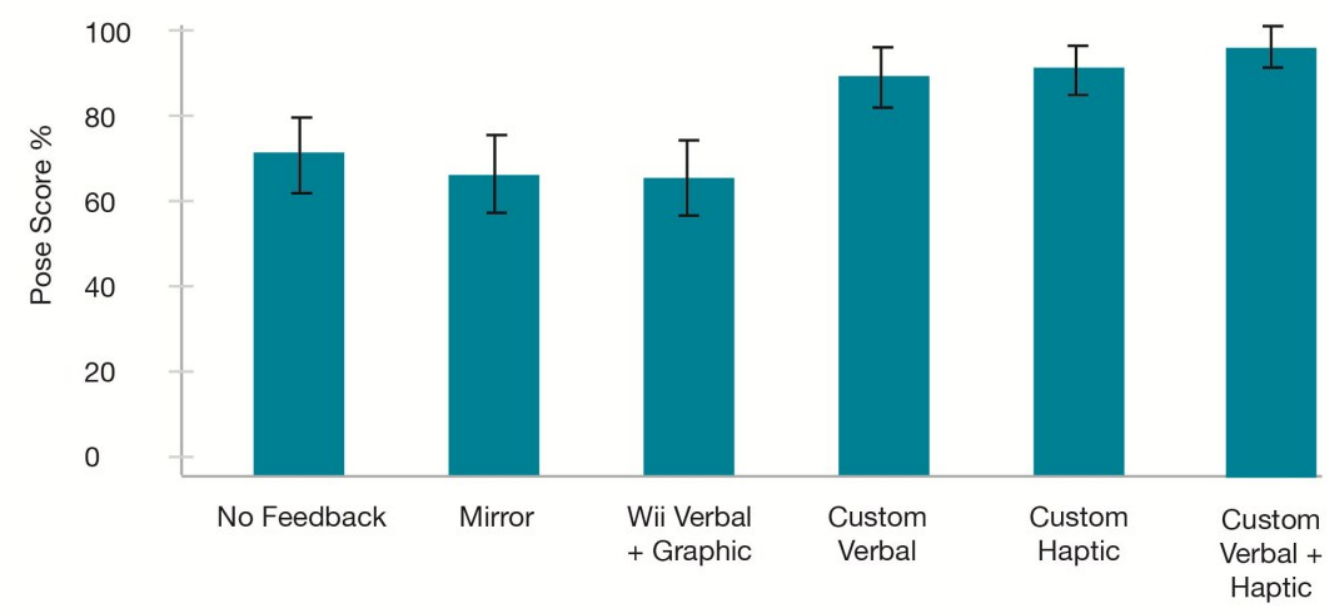

Figure 14 Pose Score (\%) of Each Feedback Type

\subsection{Results}

We analyzed the collected measures by performing a repeated measures analysis of variance (ANOVA) using feedback types (6) x performance scores.

We began by looking for an effect of feedback on pose accuracy. In this case, we predicted that the customized combination of verbal feedback and haptic feedback would illicit improved pose accuracy. Figure 14 presents the mean pose accuracy scores and standard deviation across feedback types. Here, we can already see a positive increase in pose accuracy for the three customized feedback types. Mauchly's test of Sphericity was applied to assess variance and covariance. This test was found to be significant $\left[\chi_{2}(14)=\right.$ $42.436, p=.000, \mathrm{~W}=.084]$ suggesting that the assumption of sphericity was violated and groups do not demonstrate homogeneity. The Greenhouse-Geisser correction revealed a statistically significant effect of feedback conditions on pose accuracy, $F(2.900,55.107)$ $=10.231, p<.0005$. partial $\eta^{2}=.350$. Post-hoc analysis with a Boneferroni adjustment revealed that performance significantly increased from no feedback to custom verbal 
feedback (.156 (95\% CI, .004 to .307), $p=.041)$, and from no feedback to custom haptic feedback $(.169(95 \% C I, .009$ to .329$), p=.032)$ and finally, the largest increase in

\begin{tabular}{|c|c|c|c|c|c|}
\hline & & & & & \\
\hline & & & & Sel & \\
\hline Befc & ack & & & Pos & \\
\hline Mean & SD & Mean & SD & Mean & SD \\
\hline 3.65 & 1.23 & - & - & 3.4 & 0.88 \\
\hline 4.2 & .89 & 4.25 & 0.85 & 3.9 & 1.02 \\
\hline 4.35 & 0.59 & 4.55 & 0.60 & 3.95 & 0.76 \\
\hline 4.00 & 0.86 & 4.55 & 0.51 & 3.6 & 1.1 \\
\hline 3.95 & 1.32 & 4.26 & 0.99 & 3.65 & 1.31 \\
\hline 3.85 & 0.99 & 4.55 & 0.94 & 3.70 & 1.13 \\
\hline
\end{tabular}

Table 9 Mean and Standard Deviation of Likert Pose-Pose Responses

performance from no feedback to the combination of custom verbal and haptic feedback (.184 (95\% CI, .032 to .336), $p=.010)$. Neither mirror feedback (-.061 (95\% CI, -.294 to $.173), \mathrm{p}=1.00)$, nor Wii graphic + verbal feedback (-.048 (95\% CI, -.274 to .179), $p=$ 1.00), had a significant increase in performance from no feedback. 


\subsubsection{User Feedback}

We then looked at the Likert scores participants provided after each pose to determine if there is an effect of feedback on confidence and on perceived pose accuracy (Table 9). Here, we predicted that the customized combination of verbal feedback and haptic feedback would improve confidence and also perceived pose accuracy, along side performance.

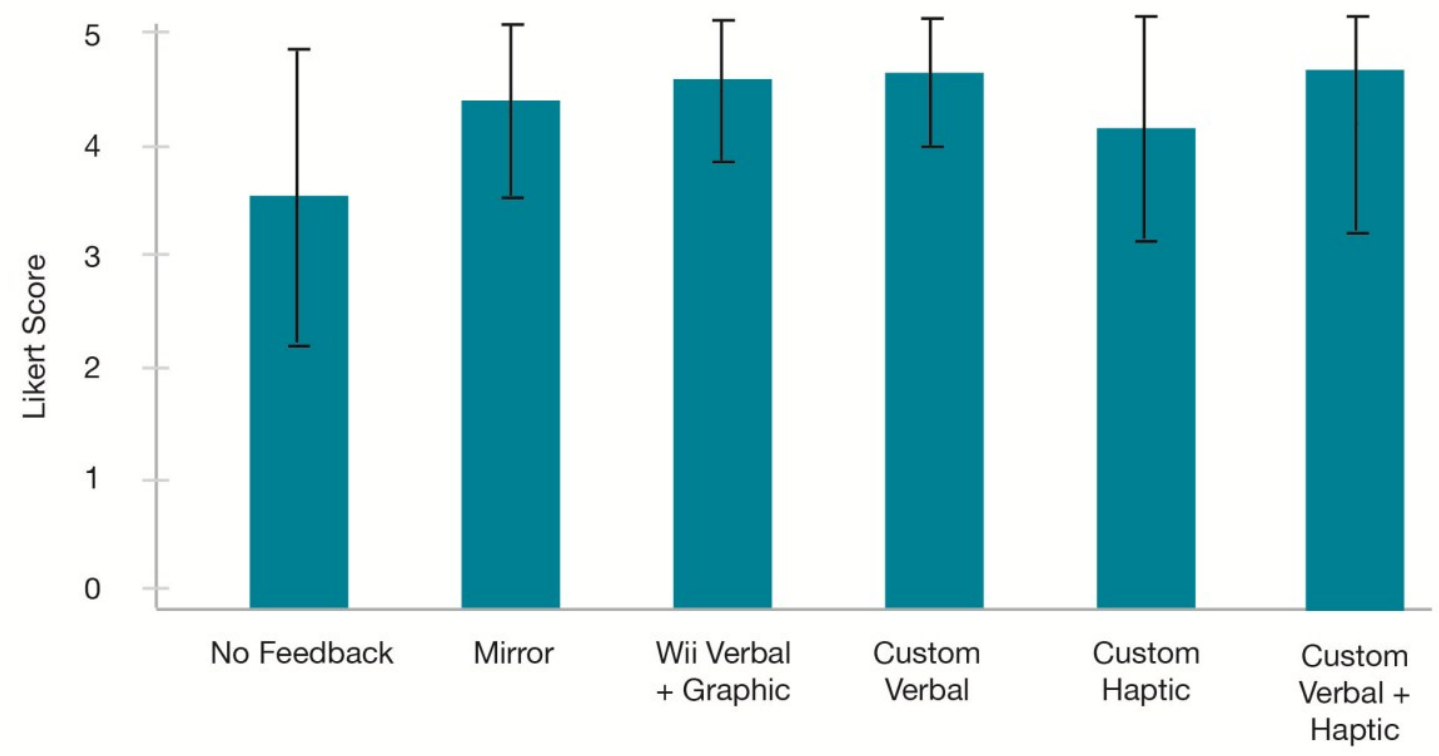

Figure 15 Mean Likert Scores for Confidence After Feedback

We began by examining confidence. Figure 15 presents the mean Likert scores and standard deviation for confidence in understanding after feedback across each feedback type. There appears to be a lot of variability, but we can see the Wii graphic + verbal, custom verbal and haptic + custom verbal feedback types excel, but it should be noted that the Wii feedback seem particularly inflated after noting the poor performance scores for that feedback type. A repeated measures ANOVA revealed a statistically significant relationship between confidence in understanding and feedback conditions, $F$ 
$(5,90)=5.671, p<.013$, partial $\eta^{2}=.240$. Pairwise post-hoc tests, with Bonferroni corrected comparisons, showed that only verbal feedback resulted in a significant increase from no feedback.

We then inspected participant self-efficacy by examining the effect of feedback types on participant-reported Likert scores of perceived pose accuracy. Analysis did not reveal a significant effect of self-reported pose accuracy on feedback type, $F(5,95)=$ $.842, p>.05$, partial $\eta^{2}=.042$.

We performed an exploratory investigation of individual participant responses. Transcribed and coded explanations for post-pose question Likert responses can be found on page 53. Categories were combined to form groups of 'negative' and 'positive' comments. Figure 16 illustrates the disparity between the three custom feedback types (Custom verbal, Custom haptic, Custom haptic/verbal) had fewer negative comments,

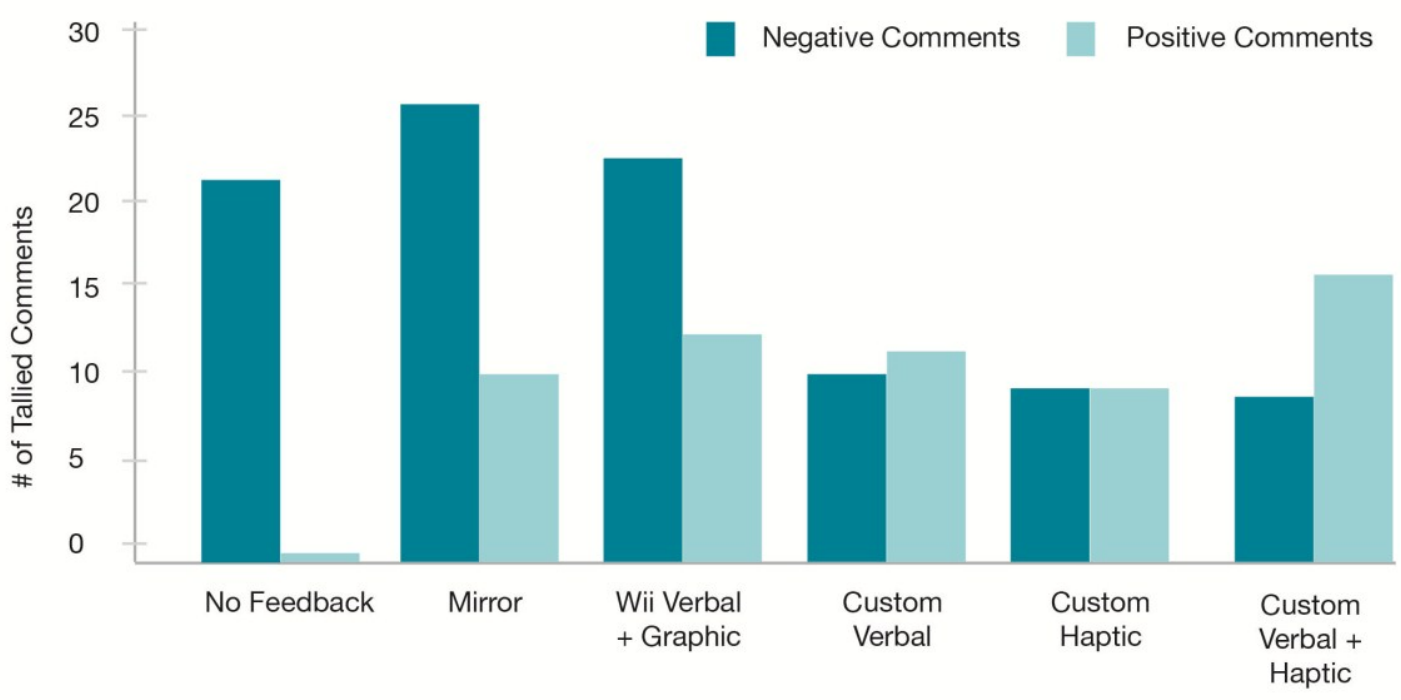

Figure 16 Grouped Negative \& Positive Comments for Feedback Types 
while custom haptic + verbal received more positive comments than any other.

Lastly, we examined the results from the exit questionnaire in Table 6. Here, we can see the participant inclination for more customized feedback types with a particular preference for custom verbal + haptic feedback.

\begin{tabular}{|c|c|c|c|c|c|c|c|c|c|}
\hline \multicolumn{2}{|c|}{ Mirror } & \multicolumn{2}{|c|}{$\begin{array}{l}\text { Wii graphic + } \\
\text { verbal }\end{array}$} & \multicolumn{2}{|c|}{ Verbal } & \multicolumn{2}{|c|}{ Haptic } & \multicolumn{2}{|c|}{$\begin{array}{c}\text { Verbal + } \\
\text { haptic }\end{array}$} \\
\hline Mean & SD & Mean & SD & Mean & SD & Mean & SD & Mean & SD \\
\hline 3.05 & 1.54 & 3.4 & 1.09 & 4.47 & 0.77 & 4.26 & 1.15 & 4.75 & 0.55 \\
\hline $\begin{array}{r}\text { Most } \\
\text { Verbal + }\end{array}$ & $\begin{array}{l}\text { Helpful } \\
\text { Haptic }\end{array}$ & $\begin{array}{r}\text { Least } \\
\mathrm{Mi}\end{array}$ & $\begin{array}{l}\text { Helpful } \\
\text { ror }\end{array}$ & & & & & & \\
\hline Mode & SD & Mode & SD & & & & & & \\
\hline 6 & 1.48 & 2 & 0.87 & & & & & & \\
\hline
\end{tabular}

Table 10 Mean and Standard Deviation of Exit Questionnaire Responses 


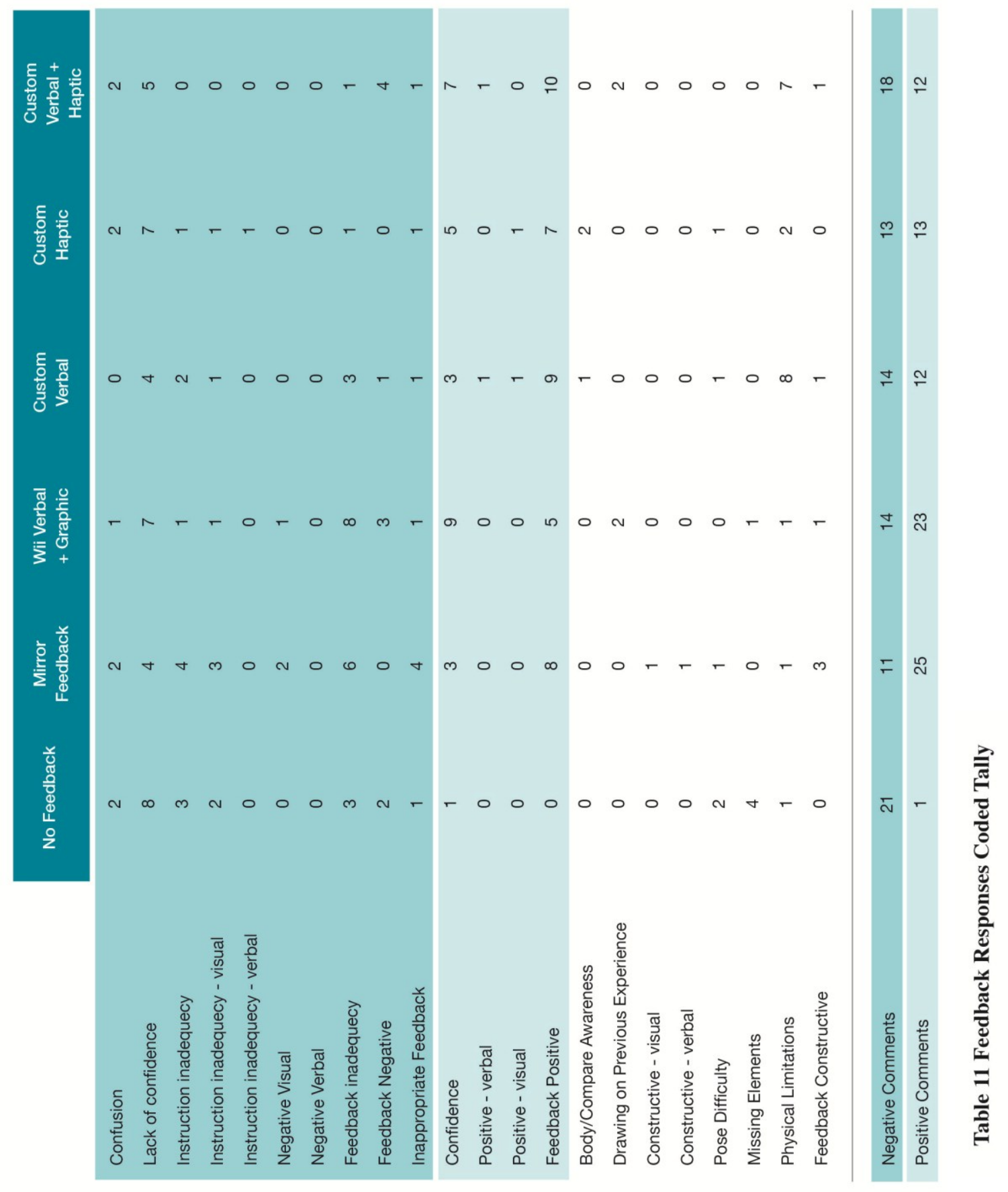




\subsection{Discussion}

As predicted, participants scored higher on pose accuracy with more customized feedback. While custom verbal and haptic feedback, individually had a significant relationship with pose accuracy, haptic + verbal feedback received the highest mean score with $95 \%$ and a standard deviation of 0.090 (H9). Participant reports found that this was not only the preferred feedback type but was also found to make participants the most confident in their understanding of the pose. These findings demonstrate that a more customized type of feedback is necessary to ensure not only performance accuracy, which is essential for an at-home rehabilitation system, but also users' confidence in their understanding and their ability. Statistical analysis also demonstrated that haptic + verbal feedback significantly increased participant confidence in understanding compared to no feedback (H10).

While mean Likert scores in confidence after receiving feedback for Wii graphic + verbal, custom verbal and custom verbal + haptic feedback were the same, it should be noted that mean pose accuracy scores for poses that received Wii feedback was observably lower than other feedback types at $72 \%, \mathrm{SD}=0.20$ (H11). This seems to suggest that the Wii feedback makes users over confident in their actions even when they are not performing poses correctly. Users do not receive the benefits of the pose while not being aware of their inaccuracies and may lead to injury if over confidence leads to over exertion.

This trend was also found in verbal accounts where Wii feedback had the most commentary regarding feedback inadequacy, but also received the highest amount of reports for confidence. We speculate this is due to participants not being clear on how to 
interpret the system's feedback but then feeling confident in pose completion once the system congratulated them on a job well done. Some participants commented on the inadequacy of the Wii feedback:

"I was confident in what I had to do but the feedback was unclear. I didn't know what to do to my body get myself into the correct position." - Participant 15

"I was only paying attention to my weight distribution and not paying attention to anything else. The system wouldn't have known if anything else is off." - Participant 3

"I could concentrate on my balance but I didn't pay attention to the rest of my body and wasn't sure if I was in the correct position" - Participant 6

In the categorized verbal responses we can see that physical limitation was noted by participants a number of times (24). While this was not under review in our testing it is our belief that users shouldn't ever feel as though they cannot perform an action, particularly in a rehabilitation scenario when the system should be better geared to a patient's abilities. The system should provide appropriate alternatives that still capture the major benefit of the pose. In a similar vein, research in the field of exergaming has explored adapting a game's difficulty to a user's heart rate [52]. Future research might explore the effect of pose alternatives and detection of user ability on confidence and pose accuracy. 


\section{Conclusions}

In this thesis, we examined elements that contribute to pose comprehension in a virtual 3D environment including occlusion and lack of visibility, learning and orientation, and feedback, in an effort to better understand what is necessary to achieve a high level of performance, confidence and motivation. With occlusion and lack of visibility, visual obstruction can prevent the viewer from obtaining visual data required to complete an accurate mental image of the object viewed. Learning and orientation refers to the need for maintaining the user's sense of orientation of self and environment in comparison to their virtual avatar and the virtual environment. This is essential in learning through visual imitation and comparison to the instructor. Finally, feedback is defined as any visual, verbal or physical criticism of an individual's actions that lead them to achieve a target. In video games, initial instruction usually demands the participant's full attention, however effective feedback has to consider the interpretation factors described above while considering the patient's context. As we have discussed, by evaluating these elements we hoped to realize an ideal visual demonstration delivery that maximizes pose understanding and user self-efficacy, determine whether supplementary modalities are important for instruction, and also determine if there is an ideal feedback delivery that promotes pose comprehension, confidence and motivation.

We evaluated these factors in two experiments. The first experiment was divided into two sections. The first section focused on visual demonstration delivery and determined that a panning view, which includes an angle that captures the greatest visible surface area of the instructor and additional angles is preferred though did not greatly impact performance. The second section focused on supplementary modalities and 
established that verbal delivery improved performance and increased user confidence while being a favoured element in demonstration. Our second experiment established that more customized and contextual modes of feedback are necessary to improve feedback and increase user comprehension and confidence. While addressing our design goals, these evaluations offer a direction and set of guidelines for designing for rehabilitation.

\subsection{Summary of Findings}

\begin{tabular}{|l|l|l|l|}
\hline \multicolumn{2}{|l|}{ Hypothesis } & Result & Significance \\
\hline Study 1: Demonstration Delivery Set 1: Visual Delivery & $\begin{array}{l}\text { H1 } \\
\text { Participants' comprehension } \\
\text { score will be best when offered } \\
\text { a single, short but continuous, } \\
\text { multi-view demonstration that } \\
\text { will ensure any obstructed or } \\
\text { occluded pose elements are } \\
\text { clarified. }\end{array}$ & $\begin{array}{l}\text { Analysis did not reveal a } \\
\text { significant relationship } \\
\text { between pose score and } \\
\text { visual demonstration } \\
\text { conditions. Hypothesis } \\
\text { not supported. }\end{array}$ & Not Significant \\
\hline H2 & $\begin{array}{l}\text { Too many additional images } \\
\text { will be visually overwhelming } \\
\text { to participants. }\end{array}$ & $\begin{array}{l}\text { While not significant, an } \\
\text { increase in mean pose } \\
\text { accuracy from 1 to 2 } \\
\text { additional images may } \\
\text { suggest further pose } \\
\text { improvement with more } \\
\text { additional visual } \\
\text { information. Hypothesis } \\
\text { not supported. }\end{array}$ & Not Significant \\
\hline
\end{tabular}




\begin{tabular}{|c|c|c|c|}
\hline $\mathrm{H} 3$ & $\begin{array}{l}\text { Participants will score lowest } \\
\text { when presented with only a } \\
\text { front view of the pose. }\end{array}$ & $\begin{array}{l}\text { While not significant, } \\
\text { participants reported that } \\
\text { the Front view instruction } \\
\text { felt inadequate. } \\
\text { Participants rated quality } \\
\text { of demonstration of the } \\
\text { Front view significantly } \\
\text { lower than other visual } \\
\text { types. Additionally, they } \\
\text { rated it the lowest in exit } \\
\text { questionnaires. } \\
\text { Hypothesis not } \\
\text { supported. }\end{array}$ & Not Significant \\
\hline $\mathrm{H} 4$ & $\begin{array}{l}\text { Participants will feel most } \\
\text { confident in their } \\
\text { understanding of poses } \\
\text { offering a single, short but } \\
\text { continuous, multi-view } \\
\text { demonstration. }\end{array}$ & $\begin{array}{l}\text { Analysis did not reveal a } \\
\text { significant relationship } \\
\text { between confidence and } \\
\text { visual demonstration } \\
\text { conditions. Hypothesis } \\
\text { not supported. }\end{array}$ & Not Significant \\
\hline H5 & $\begin{array}{l}\text { Participants will feel least } \\
\text { confident when provided only } \\
\text { a single view of a pose. }\end{array}$ & $\begin{array}{l}\text { Analysis did not reveal a } \\
\text { significant relationship } \\
\text { between confidence and } \\
\text { visual demonstration } \\
\text { conditions. Hypothesis } \\
\text { not supported. }\end{array}$ & Not Significant \\
\hline \multicolumn{4}{|c|}{ Study 1: Demonstration Delivery $\quad$ Set 2: Verbal Delivery } \\
\hline H6 & $\begin{array}{l}\text { Participants would score higher } \\
\text { if they were provided with } \\
\text { verbal description of the pose } \\
\text { in addition to visual. }\end{array}$ & $\begin{array}{l}\text { We discover that there is, } \\
\text { in fact, a statistically } \\
\text { significant effect of } \\
\text { verbal conditions on pose }\end{array}$ & Significant \\
\hline
\end{tabular}




\begin{tabular}{|c|c|c|c|}
\hline & & $\begin{array}{l}\text { score. Hypothesis } \\
\text { supported. }\end{array}$ & \\
\hline $\mathrm{H} 7$ & $\begin{array}{l}\text { Participants would rate } \\
\text { confidence higher if they were } \\
\text { provided with verbal } \\
\text { description of the pose in } \\
\text { addition to visual. }\end{array}$ & $\begin{array}{l}\text { We discover that there is } \\
\text { a statistically significant } \\
\text { effect of verbal } \\
\text { conditions on confidence, } \\
\text { and also quality of } \\
\text { demonstration. } \\
\text { Hypothesis supported. }\end{array}$ & $\begin{array}{l}\text { Confidence: } \\
\text { Significant } \\
\text { Quality of } \\
\text { Demonstration: } \\
\text { Significant }\end{array}$ \\
\hline H8 & $\begin{array}{l}\text { Participants would score lower } \\
\text { when only provided visual } \\
\text { demonstration (no verbal). }\end{array}$ & $\begin{array}{l}\text { We discover that there is, } \\
\text { in fact, a statistically } \\
\text { significant effect of } \\
\text { verbal conditions on pose } \\
\text { score. Mean Pose scores } \\
\text { were significantly lower } \\
\text { for non-verbal } \\
\text { conditions. Hypothesis } \\
\text { supported. } \\
\text { Participants also } \\
\text { preferred instruction with } \\
\text { verbal delivery and rated } \\
\text { instruction without it } \\
\text { 'inadequate' }\end{array}$ & Significant \\
\hline \multicolumn{4}{|c|}{ Study 2: Feedback } \\
\hline H9 & $\begin{array}{l}\text { Participants would perform } \\
\text { best when provided with } \\
\text { customized verbal and haptic } \\
\text { feedback that replicated } \\
\text { feedback delivery offered by a } \\
\text { physiotherapist. }\end{array}$ & $\begin{array}{l}\text { The Greenhouse-Geisser } \\
\text { correction revealed a } \\
\text { statistically significant } \\
\text { effect of feedback } \\
\text { conditions on pose } \\
\text { accuracy. Hypothesis } \\
\text { supported. }\end{array}$ & Significant \\
\hline
\end{tabular}




\begin{tabular}{|c|c|c|c|}
\hline & \multicolumn{3}{|c|}{$\begin{array}{l}\text { Post-hoc analysis with a Boneferroni adjustment revealed that performance } \\
\text { significantly increased from no feedback to verbal feedback and from no feedback } \\
\text { to haptic feedback and finally, the largest increase in performance from no } \\
\text { feedback to the combination of verbal and haptic feedback. }\end{array}$} \\
\hline H10 & $\begin{array}{l}\text { Participants would feel most } \\
\text { confident in their pose } \\
\text { understanding when provided } \\
\text { with customized verbal and } \\
\text { haptic feedback that replicated } \\
\text { feedback delivery offered by a } \\
\text { physiotherapist. }\end{array}$ & $\begin{array}{l}\text { The analysis revealed a } \\
\text { statistically significant } \\
\text { relationship between } \\
\text { confidence in } \\
\text { understanding and } \\
\text { feedback conditions. } \\
\text { Hypothesis supported. }\end{array}$ & Significant \\
\hline H11 & $\begin{array}{l}\text { Wii feedback with balance } \\
\text { board would improve } \\
\text { confidence but not } \\
\text { performance score. }\end{array}$ & $\begin{array}{l}\text { While confidence was } \\
\text { still very high for the Wii } \\
\text { feedback condition, } \\
\text { participants actually } \\
\text { performed significantly } \\
\text { lower than the verbal + } \\
\text { haptic feedback type, } \\
\text { demonstrating an } \\
\text { imposed over-confidence } \\
\text { from the Wii system. } \\
\text { This trend was also } \\
\text { reflected in the coded } \\
\text { responses where } \\
\text { confidence was tallied } \\
\text { high for Wii feedback. } \\
\text { Conversely, instruction } \\
\text { inadequacy and lack of } \\
\text { confidence in the Wii } \\
\text { feedback were much } \\
\text { higher than other } \\
\text { feedback types. }\end{array}$ & \\
\hline
\end{tabular}




\subsection{Design Recommendations}

The evaluation of these factors can be summarized into the following design recommendations for future research and design of interactive gaming systems for athome rehabilitation:

1. Multiple Visual Angle Demonstration: When demonstrating a pose, we recommend that users be provided with multiple angles of a demonstration to help them resolve the visual information they require to understand a pose. Additional angles can provide more points of reference for the user to check their understanding or self-positioning. Panning can offer a seamless range of view without imposing a cognitive increase with a visual angle switch. Although there were no significant differences between pose accuracy or confidence and visual delivery methods, the inclusion of a wide view that incorporated the largest amount of visible surface area of the instructor, a panning view and also simultaneous additional angles were found to be preferred elements by users.

2. Multi-modal Demonstration: We observed that participants required verbal or clear descriptive language about what their body should be doing, where to feel the stretch, when to breathe etc., in order to complete their mental model of the pose but also to feel confident in their understanding on the pose being executed. Descriptive verbal delivery was found to have a significant relationship with pose accuracy and confidence in understanding. Instruction without verbal description was found to feel inadequate and lead to a lack in confidence. Some participants expressed the importance of 
analogies to help relate to the pose more effectively. This can help connect with users and can contribute to engagement.

3. Clear Orientation Environment: When providing a visual demonstration we recommend that instruction begins by establishing a clear orientation environment. This may include the ability to face the instructor away or towards the user or simple visual cues like a yoga mat. This allows the user to mentally clarify the relationship between their own body and the instructor. We observed that several users would miss an entire demonstration because they were cognitively engrossed in interpreting which hand or foot to use. Establishing a clear orientation environment becomes especially important with complicated poses.

4. Verbal Delivery as Supplementary: Relating to the previous recommendation, when demonstrating a pose, we suggest that verbal delivery remain a supplementary modality to visual delivery. We observed that the use of direction (i.e., left and right) in description often lead to confusion. Visual delivery should be used as a point of reference or imitation while verbal delivery provides analogy, unobservable detail and establishes rapport with the user. Haptic feedback can also assist with directional instruction.

5. Custom, Contextual feedback: Though we can really only evaluate currently available fitness and training games that offer feedback, this feedback was found to be inappropriate for more performance focused activities like training and rehabilitation. When offering feedback for these 
situations, we recommend providing users with customized, contextual feedback to help them resolve errors. Custom, contextual feedback offers a direct solution to inaccuracy rather than just demonstrating inaccuracy and allowing the user to determine a solution. Custom, contextual feedback types, including verbal, haptic and a combination of these, were found to have a significant relationship with pose performance and were also highly preferred to commercially available, Wii system feedback or self-regulated modes of feedback such as a mirror or avatar of one's self.

6. Multi-Modal Feedback: Speaking further to the point of contextual feedback, we recommend that feedback be provided in a modality that compliments the orientation of the action. It is likely the user is mid-action when receiving feedback, in these cases, the system should adapt it is feedback modality to accommodate the user - provide haptic or verbal feedback if the user's eyes are averted, for example. It was noted that participants rated modalities that were ill-matched to the pose worse than more appropriate ones. Some participants did not even take advantage of the feedback provided because they weren't able to see it from the position they were in. In some cases, participants performed as though they had no feedback at all if they were provided with an ill-matched modality and even said they did not use or notice the feedback.

7. Understand \& Adapt For Capability: Especially for training and rehabilitation, the system's awareness of the user's capabilities is essential to ensuring poses are performed accurately and without injury. For this 
reason we recommend that the system be aware of user ability and provide alternative actions that still address the benefit of the pose without overexerting or discouraging the user. During analysis we found that participants often commented on their physical limitations when attempting the poses. Not feeling capable or able to complete a pose can lead to noncompliance. Providing participants with alternatives, that still address the gross benefit of the action, yet still offer a challenge, may make users feel confident while still performing the pose correctly, without risk of injury and working towards recovery.

While we recognize that many of these recommendations are not currently realizable and may still require a lot of technical exploration, we feel that by exploring user needs and submitting possible solutions we can begin the iterative journey to a well-designed application that exceeds the needs of the user.

\subsection{Limitations and Future Work}

This thesis only addresses some of the many factors that contribute to action comprehension. The goal of this thesis was to begin to build a foundational set of design guidelines for further research in the area where none exists.

In the visual delivery section we chose a very general set of delivery types to reveal patterns that could be later explored. We feel we accomplished this. While we did not find a significant relationship between the deliveries we chose and pose accuracy future research in interactive gaming demonstration might assess the impact of varying 
amounts of visual information, including more than 2 additional angles, on performance and cognitive load and what that means for user confidence.

Though we did choose delivery types that features panning and angle jumping to evaluate differences, further research would explore the impact panning vs. angle jumping and the max and min angle jump on response time, pose accuracy and confidence. This could shed more light on optimal pan angles and length and how this affects the user's cognitive load and performance level.

The individual responses from the users also shed light on avenues for future exploration including adapting for ability, establishing an orientation environment and the effects of system latency.

Beyond elements of these studies, exploration of learning styles and adapting for different user needs could also enrich the application of guidelines for rehabilitation in gaming systems. 


\section{References}

1. Aarhus, R., Groenvall, E., Larsen, S.B., and Wollsen, S. Turning training into play: Embodied gaming, seniors, physical training and motivation. Gerontechnology 10, 2 (2011), 120-120.

2. Alankus, G., Lazar, A., and May, M. Towards customizable games for stroke rehabilitation. Proceedings of CHI 2010: Therapy and Rehabilitation, (2010), 2113-2122.

3. American Academy of Orthopedic Surgeons. Sprained Ankle. 2012. http://orthoinfo.aaos.org/topic.cfm?topic=a00150.

4. Anderson, F., Annett, M., and Bischof, W.F. Lean on Wii: physical rehabilitation with virtual reality Wii peripherals. Studies in health technology and informatics 154, (2010), 229-34.

5. Billenstein Schriver, N. and Engelsrud, G. Rehabilitation of a Knee Injury: Tensions Between Standard Exercises and Lived Experiences. Journal of Sport \& Social Issues 35, 1 (2011), $22-32$.

6. Billis, A.S., Konstantinidis, E.I., Mouzakidis, C., Tsolaki, M.N., Pappas, C., and Bamidis, P.D. A Game-Like Interface for Training Seniors ’ Dynamic Balance and Coordination. Proceedings of MEDICON 2010, (2010), 691-694.

7. Brown, E. and Cairns, P. A Grounded Investigation of Game Immersion. Extended abstracts of the 2004 conference on Human factors and computing systems - CHI '04, ACM Press (2004), 1297.

8. Burdea, G. Virtual Rehabilitation- Benefits and Challenges. Methods of Information in Medicine 200342,5 (2003), 519-523.

9. Burke, J.W., McNeill, M.D.J., Charles, D.K., Morrow, P.J., Crosbie, J.H., and McDonough, S.M. Optimising engagement for stroke rehabilitation using serious games. The Visual Computer 25, 12 (2009), 1085-1099.

10. Canada Immigration. New list of high demand occupations for canadian immigration. 2013. http:/www.brilliancyimmigration.com/new-list-of-high-demand-occupations-for-canadianimmigration.

11. Chang, C., Lange, B., and Zhang, M. Towards pervasive physical rehabilitation using Microsoft Kinect. 2012 6th International Conference on Pervasive Computing Technologies for Healthcare (PervasiveHealth) and Workshops, (2012), 159-162.

12. Courneya, K.S. and McAuley, E. Cognitive mediators of the social influence-exercise adherence relationship: a test of the theory of planned behavior. Journal of behavioral medicine 18, 5 (1995), 499-515.

13. Crook, P., Rose, M., Salmon, P., Stott, R., Peters, S., and Stanley, I. Adherence to group exercise: Physiotherapist-led experimental programmes. Physiotherapy 84, 8 (1998), 366-372.

14. Cross, M.J. Anterior Cruciate Ligament Injuries: Treatment and Rehabilitation. Encyclopedia of Sports Medicine and Science, 1998.

15. Csikszentmihalyi, M. Flow: The psychology of optimal performance. Harper and Row, New York, 1990 . 
16. Daems, A. and Verfaillie, K. Viewpoint-dependent priming effects in the perception of human actions and body postures. Visual Cognition 6, 6 (1999), 665-693.

17. Daniel, D. and Stone, M. Fate of the ACL-injured patient a prospective outcome study. The American Journal of Sports Medicine 22, 5 (1994), 632-644.

18. Decker, J., Li, H., Losowyj, D., and Prakash, V. Wiihabilitation: rehabilitation of wrist flexion and extension using a wiimote-based game system. 2009.

http://www.soe.rutgers.edu/sites/default/files/gset/Wii.pdf.

19. Deutsch, J.E., Borbely, M., Filler, J., Huhn, K., and Guarrera-Bowlby, P. Use of a low-cost, commercially available gaming console (Wii) for rehabilitation of an adolescent with cerebral palsy. Physical therapy 88, 10 (2008), 1196-207.

20. Dishman, R.K. and Ickes, W. Self-motivation and adherence to therapeutic exercise. Journal of behavioral medicine 4, 4 (1981), 421-38.

21. Dishman, R.K. Erercise Adherence: Its Impact on Public Health. 1993.

22. EHealthMD. ACL Tears. 2013. http://ehealthmd.com/acl-tears/what-anterior-cruciateligament\#axzz2SX2c9LKJ.

23. Epstein, L.H. and Cluss, P. a. A Behavioral Medicine Perspective on Adherence to Long-Term Medical Regimens. Journal of consulting and clinical psychology 50, 6 (1982), 950-71.

24. Flores, E., Tobon, G., Cavallaro, E., Cavallaro, F.I., Perry, J.C., and Keller, T. Improving patient motivation in game development for motor deficit rehabilitation. Proceedings of the 2008

International Conference in Advances on Computer Entertainment Technology - ACE '08, (2008), 381.

25. Fraser, S.N. and Spink, K.S. Examining the role of social support and group cohesion in exercise compliance. Journal of behavioral medicine 25, 3 (2002), 233-49.

26. Freitas, D.Q., Da Gama, A., Figueiredo, L., and Chaves, T.M. Development and Evaluation of a Kinect Based Motor Rehabilitation Game. Proceedings of SBGames 2012, (2012), 144-153.

27. Friedrich, M., Gittler, G., Halberstadt, Y., Cermak, T., and Heiller, I. Combined exercise and motivation program: effect on the compliance and level of disability of patients with chronic low back pain: a randomized controlled trial. Archives of physical medicine and rehabilitation 79, 5 (1998), 475-87.

28. Da Gama, A., Chaves, T., Figueiredo, L., and Teichrieb, V. Guidance and Movement Correction Based on Therapeutics Movements for Motor Rehabilitation Support Systems. 2012 14th Symposium on Virtual and Augmented Reality, Ieee (2012), 191-200.

29. Gerling, K. and Schild, J. Exergame design for elderly users: the case study of SilverBalance. Proceedings of ACE 2010, (2010), 66-69.

30. Gerling, K. and Schulte, F. Designing and Evaluating Digital Games for Frail Elderly Persons. Proceedings of ACE 2011, (2011). 
31. Gerling, K.M., Livingston, I.J., Nacke, L.E., and Mandryk, R.L. Full-Body Motion-Based Game Interaction for Older Adults. Proceedings of CHI 2012: 30th international conference on Human factors in computing systems, (2012).

32. Glaser, B. and Strauss, A. The Discovery of Grounded Theory Method: Strategies for qualitative research. Transaction Books, 1967.

33. Griffiths, M. Can videogames be good for your health? Journal of health psychology 9, 3 (2004), 339-44.

34. Haynes, R. Brian, and D.L.S. Compliance in health care. 1979.

35. Hoogen, W. Van Den, Ijsselsteijn, W., and Kort, Y. De. Yes Wii Can! using digital games as a rehabilitation platform after stroke - the role of social support. Virtual Rehabilitation International Conference, 2009, (2009), 195.

36. Hupperets, M.D.W., Verhagen, E. a L.M., and Mechelen, W. V. Effect of unsupervised home based proprioceptive training on recurrences of ankle sprain: randomised controlled trial. BMJ 2009;339: b2684., (2009), b2684.

37. Härkäpää K, Järvikoski A, Mellin G, H.H. A controlled study on the outcome of inpatient and outpatient treatment of low back pain. Part I. Pain, disability, compliance, and reported treatment benefits three months after treatment. Scandinavian journal of rehabilitation medicine 21, 2 (1989), 81 .

38. Ice, R. Long-term compliance. Journal of the American Physical Therapy Association 65, 12 (1985), 1832-1839.

39. Jack, K., McLean, S.M., Moffett, J.K., and Gardiner, E. Barriers to treatment adherence in physiotherapy outpatient clinics: a systematic review. Manual therapy 15, 3 (2010), 220-8.

40. Jette, A.M. Improving patient cooperation with arthritis treatment regimens. Arthritis \& Rheumatism 25, 4 (1982), 447-453.

41. Johnston, H. and Whitehead, A. Pose presentation for a dance-based massively multiplayer online exergame. Entertainment Computing 2, 2 (2011), 89-96.

42. Jung, Y., Li, W., Gladys, C., and Lee, K.M. Games for a Better Life : Effects of Playing Wii Games on the Well-Being of Seniors in a Long-Term Care Facility. Proceedings of the Sixth Australasian Conference on Interactive Entertainment, (2009), 0-5.

43. Kelly, R.B., Zyzanski, S.J., and Alemagno, S. a. Prediction of motivation and behavior change following health promotion: role of health beliefs, social support, and self-efficacy. Social science \& medicine (1982) 32, 3 (1991), 311-20.

44. Kifayat, K., Fergus, P., Cooper, S., and Merabti, M. Body Area Networks for Movement Analysis in Physiotherapy Treatments. 2010 IEEE 24th International Conference on Advanced Information Networking and Applications Workshops, Ieee (2010), 866-872.

45. Kim, K., Bolton, J., Girouard, A., Cooperstock, J., and Vertegaal, R. TeleHuman: effects of 3d perspective on gaze and pose estimation with a life-size cylindrical telepresence pod. CHI ' 12 
Proceedings of the SIGCHI Conference on Human Factors in Computing Systems, (2012), 25312540.

46. Klemmer, S.R., Hartmann, B., and Takayama, L. How Bodies Matter : Five Themes for Interaction Design. Proceedings of the 6th conference on Designing Interactive systems, (2006), 140-149.

47. Lange, B., Flynn, S., and Rizzo, A. Initial usability assessment of off-the-shelf video game consoles for clinical game-based motor rehabilitation. Physical Therapy Reviews 14, 5 (2009), 355-363.

48. Leder, R. and Azcarate, G. Nintendo Wii remote for computer simulated arm and wrist therapy in stroke survivors with upper extremity hemipariesis. Virtual Rehabilitation 10, 2 (2008), 4244.

49. Löwgren, J. interaction aesthetics. (2009), 1-18.

50. Martin, J.E. and Dubbert, P.M. Exercise applications and promotion in behavioral medicine: current status and future directions. Journal of consulting and clinical psychology 50, 6 (1982), 1004-17.

51. Martin-Moreno, J., Ruiz-Fernandez, D., Soriano-Paya, A., and Jesus Berenguer-Miralles, V. Monitoring 3D movements for the rehabilitation of joints in physiotherapy. Conference proceedings : ... Annual International Conference of the IEEE Engineering in Medicine and Biology Society. IEEE Engineering in Medicine and Biology Society. Conference 2008, (2008), 4836-9.

52. Masuko, S. A Fitness Game Reflecting Heart Rate. Advances in Computer Entertainment Technology 53, (2006), 3-5.

53. Matarazzo, I., Weiss, S., Herd, J., and Oldridge, N. Adherence to adult exercise fitness programs. In Behavioral Health: A Handbook of Health Enhancement and Disease Prevention. 1984, 467487.

54. Mclean, S., Burton, M., Bradley, L., and Littlewood, C. Interventions for enhancing adherence with physiotherapy : a systematic review. Manual therapy 15, 6 (2010), 514-521.

55. Mendoca, K. DESENVOLVIMENTO E VALIDAÇÃO DE UM INSTRUMENTO DE MEDIDA. Revista Brasileira de Fisioterapia 11, 5 (2007), 369-76.

56. Mitchell, R. Kinesthetic- Visual Matching and the Self- Concept as Explanations of Mirror- SelfRecognition. Journal for the theory of social behaviour 27, 1 (1997), 17-39.

57. Mueller, F.F., Edge, D., Vetere, F., et al. Designing Sports : A Framework for Exertion Games. Proceedings of CHI 2011, (2011).

58. Negrete, M.A., Matamoros, M., Olveres, J., et al. Nintendo Wii Remote and Nunchuck as a wireless data subsystem for digital acquisition of analog physiologic data relevant to motor rehabilitation after stroke. 2009 Pan American Health Care Exchanges, (2009), 130-131.

59. Nintendo. Wii Fit Plus. 2009. http://wiifit.com/training/yoga.html.

60. Rainville, J.M., Sobel, J.B.M., Hartigan, C.M., and Wright, A.M. The Effect of Compensation Involvement on the Reporting of Pain and Disability by Patients Referred for Rehabilitation of Chronic Low Back Pain. Spine 22, 17 (1997), 2016-2024. 
61. Rand, D., Kizony, R., and Weiss, P.T.L. The Sony PlayStation II EyeToy: low-cost virtual reality for use in rehabilitation. Journal of neurologic physical therapy : JNPT 32, 4 (2008), 155-63.

62. Rizzolatti, G., Fogassi, L., and Gallese, V. Neurophysiological mechanisms underlying the understanding and imitation of action. Nature Reviews | Neuroscience 2, September (2001), 1-10.

63. Sallis, J.F. Potential vs actual benefits of exergames. Archives of pediatrics \& adolescent medicine 165, 7 (2011), 667-9.

64. Schönauer, C., Pintaric, T., and Kaufmann, H. Full body interaction for serious games in motor rehabilitation. Proceedings of the 2nd Augmented Human International Conference on - AH '11, ACM Press (2011), 1-8.

65. Service Canada. Physiotherapists. job futures, 2013.

http://www.servicecanada.gc.ca/eng/qc/job_futures/statistics/3142.shtml.

66. Sinclair, J., Hingston, P., Street, B., and Lawley, M. Exergame development using the dual flow model. Proceedings of the Sixth Australasian Conference on Interactive Entertainment, (2009).

67. Skelly, a. H., Marshall, J.R., Haughey, B.P., Davis, P.J., and Dunford, R.G. Self-Efficacy and Confidence in Outcomes as Determinants of Self-Care Practices in Inner-City, African-American Women with Non-Insulin-Dependent Diabetes. The Diabetes Educator 21, 1 (1995), 38-46.

68. Sluijs, E. and Kuijper, E. Problems patients encounter in educating patients [in Dutch]. Nederlad Tijdrchnp m r Fysiotherapie 100, (1990), 128-132.

69. Sluijs, E.M., Kok, G.J., and Zee, J. Van Der. Correlates of Exercise Compliance in Physical Therapy. Physical Therapy 73, 11 (1993), 771-782.

70. Sluijs, Emmy M., Jouke van der Zee, and G.J.K. Differences between physical therapists in attention paid to patient education. Physiotherapy Theory and Practice 9, 2 (1993), 103-118.

71. Sparks, D., Chase, D., and Coughlin, L. Informatics curio Wii have a problem : a review of selfreported Wii related injuries. November 2008 (2009), 55-58.

72. Svarstad, B.L. Physician-patient communication and patient conformity with medical advice. In The growth of bureaucratic medicine. 1976, 243.

73. Sveistrup, H. Motor rehabilitation using virtual reality. Journal of neuroengineering and rehabilitation 1, 1 (2004), 10.

74. Sweetser, P. and Wyeth, P. GameFlow: a model for evaluating player enjoyment in games. Computers in Entertainment (CIE) 3, 3 (2005), 1-24.

75. Tanaka, K., Parker, J., and Baradoy, G. A comparison of exergaming interfaces for use in rehabilitation programs and research. Loading... 6, 9 (2012), 69-81.

76. Tao, Y. and $\mathrm{Hu}, \mathrm{H}$. Building a visual tracking system for home-based rehabilitation. Proc. of the 9th Chinese Automation and Computing ..., September (2003), 343-448. 
77. Taylor, M.J.D., McCormick, D., Shawis, T., Impson, R., and Griffin, M. Activity-promoting gaming systems in exercise and rehabilitation. The Journal of Rehabilitation Research and Development 48, 10 (2011), 1171.

78. Thyrion, C. and Roll, J. Perceptual integration of illusory and imagined kinesthetic images. The Journal of Neuroscience 29, 26 (2009), 8483-8492.

79. Ubisoft. Your Shape: Fitness Evolved. 2011.

80. Whiting, N.D. The Role of Yoga Therapy in Knee Rehabilitation. 16, 16 (2006), 2006.

81. Wilson, P.M., Rodgers, W.M., Carpenter, P.J., Hall, C., Hardy, J., and Fraser, S.N. The relationship between commitment and exercise behavior. Psychology of Sport and Exercise 5, 4 (2004), 405421.

82. Yong Joo, L., Soon Yin, T., Xu, D., et al. A feasibility study using interactive commercial off-theshelf computer gaming in upper limb rehabilitation in patients after stroke. Journal of rehabilitation medicine 42, 5 (2010), 437-41.

83. Zurich, R.W., Fabra, U.P., and Technologies, M.I. The effect of social gaming in virtual reality based rehabilitation of stroke patients. Proceedings of the International Conference on Virtual Rehabilitation, (2011), 1-2. 


\section{Appendices}

\section{Appendix A Study Scripts}

\section{A.1 Demonstration Delivery Test Script}

Hi, _. My name is Monica, and I'm going to be walking you through this session today.

Before we begin, I'm just going to let you know a bit about the study and what you will be asked to do. The purpose of this study is to assess pose comprehension with and without feedback on interactive video game consoles for at-home physiotherapy conditions. There are two parts to this study - Today we will just be looking at demonstration delivery. We will ask you to watch a virtual instructor perform an exercise pose. After each exercise we will ask you, with the use of a flexible mannequin, to replicate the pose as accurately as you can and answer a few questions about your performance. Be mindful that the manikin has limitations - if you can't get the manikin into the exact pose you want, get it as close as possible and explain to me what the manikin wasn't able to accomplish. Following this, you will be asked to fill out a short exist questionnaire. The whole test should take no more than 60 minutes. If you need a break at any point, just let me know. The data collected in this experiment is strictly confidential. Your identity will not be associated with the responses you provide. Only the researchers will have access to the data - your identity will not be linked in any way to the data. If at any point during the study you wish to withdraw you can do so. Just let me know. If you decide to withdraw, your responses will be destroyed. After completing 
the exit questionnaire you will not able to withdraw your responses anymore since the researchers have no way of linking your responses to your identity.

If you would, I'm going to ask you to sign an informed consent form for us. It just says that you understand everything that I've just said to you. Do you have any questions so far? OK. Before we start to play, I'd like to ask you just a few quick questions.

QUESTIONS WILL BE ASKED AFTER EACH POSE.

On a scale from $1-5$, where 1 is not at all confident and 5 is very confident; how confident did you feel about your understanding of the pose?

On a scale from 1-5, where 1 is not at all helpful and 5 is very helpful, how would you rate the helpfulness and clarity of the pose demonstration? Why?

On a scale from $1-5$, where 1 is totally inaccurate and 5 is totally accurate, how accurately do you feel you executed the pose demonstrated? Why? 


\section{A.2 Feedback Test Script}

$\mathrm{Hi}$, . My name is Monica, and I'm going to be walking you through this session today.

Before we begin, I'm just going to let you know a bit about the study and what you will be asked to do. The purpose of this study is to assess pose comprehension with and without feedback on interactive video game consoles for at-home physiotherapy conditions There are two parts to this study - Today we will just be looking at Feedback. We will ask you to watch a virtual instructor perform an exercise pose. You will be shown the demonstration a second time at which point you will be asked replicate the pose to the best of your abilities. A Researcher will give you various types of feedback following each attempt to help you correct the pose if you happen to be a bit off. Following this you will be asked a few questions about your performance. Once you have completed the pose set, you will be asked to fill out a short exist questionnaire. The whole test should take no more than 60 minutes. If you need a break at any point, just let me know. To be explicit, we will be assessing the effectiveness of 'haptic' or touch feedback mechanisms as a method of feedback for use in at-home rehabilitation. To replicate the use of touch feedback the moderator will gently tap the location of incorrect positioning. If you are uncomfortable with this you can choose not to participate in the study. The data collected in this experiment is strictly confidential. Your identity will not be associated with the responses you provide. Only the researchers will have access to the data - your identity will not be linked in any way to the data.

If at any point during the study you wish to withdraw you can do so. Just let me know. If you decide to withdraw, your responses will be destroyed. After completing the exit 
questionnaire you will not able to withdraw your responses anymore since the researchers have no way of linking your responses to your identity. If you would, I'm going to ask you to sign an informed consent form for us. It just says that you understand everything that I've just said to you. Do you have any questions so far?

OK. Before we start to play, I'd like to ask you just a few quick questions.

\section{QUESTIONS WILL BE ASKED AFTER EACH POSE.}

On a scale from $1-5$, where 1 is not at all confident and 5 is very confident, how confident did you feel about your understanding of the pose before you began executing it?

On a scale from $1-5$, where 1 is not at all confident and 5 is very confident, how confident did you feel about your understanding of the pose once you were provided with feedback?

On a scale from 1-5, where 1 is totally inaccurate and 5 is totally accurate, how accurately do you feel you executed the pose demonstrated? 


\section{Appendix B Pre-test Questions}

TEST \# Participant

Age:

How often do you play video or mobile phone games in an average week?

Never

1-2 hours

3-6 hours

$7+$

Would you consider yourself in good physical health?

Yes Somewhat above average Average Somewhat below average No

Have you ever participated in a fitness or yoga class?

Yes/no

how often, if ever, do you practice yoga or go to fitness classes?

Never

I used to but don't anymore.

I've been to a couple

1-3 times a month

1-2 times a week

3-5 times a week 


\section{Appendix C Exit Questionnaires}

\section{C.1 Demonstration Delivery Test Exit Questionnaire}

Which type of feedback did you find most helpful?
$\square \quad$ Only front view
$\square \quad$ Front then switch to wide view
$\square$ Front, Pan to Wide view
$\square$ Front, Pan to Wide view +1 additional view
$\square \quad$ Front, Pan to Wide view +2 additional view

Which type of feedback did you find least helpful?
$\square$ Only front view
$\square \quad$ Front then switch to wide view
$\square \quad$ Front, Pan to Wide view
$\square \quad$ Front, Pan to Wide view +1 additional view
$\square$ Front, Pan to Wide view +2 additional view

On a scale from $1-5$, where 1 is not at all helpful and 5 is very helpful, how would you rate the helpfulness the addition of a wide view?
1
2
3
4
5

not helpful

very helpful

On a scale from 1-5, where 1 is not at all helpful and 5 is very helpful, how would you rate the helpfulness the addition of a panning view?

$\begin{array}{ccccc}1 & 2 & 3 & 4 & 5 \\ \text { not helpful } & & & & \text { very helpful }\end{array}$

On a scale from $1-5$, where 1 is not at all helpful and 5 is very helpful, how would you rate the helpfulness of a single additional view?
1
2
3
4
5

not helpful

very helpful

On a scale from 1-5, where 1 is not at all helpful and 5 is very helpful, how would you rate the helpfulness of multiple additional views?
1
2
3
4
5
not helpful
very helpful 
On a scale from 1-5, where 1 is not at all helpful and 5 is very helpful, how would you rate the helpfulness the verbal description of the pose during demonstration?

1

not helpful
2

3

3

very helpful

\section{C.2 Feedback Test Exit Questionnaire}

FEEDBACK: Exit Survey 

Which type of feedback did you find most helpful?
$\square \quad$ No feedback
$\square \quad$ Mirror as feedback
$\square \quad$ Visual onscreen graphic feedback
$\square \quad$ Haptic or touch feedback
$\square \quad$ Custom verbal feedback
$\square$ Customer verbal + haptic/touch feedback
Which type of feedback did you find least helpful?
$\square \quad$ No feedback
$\square \quad$ Mirror as feedback
$\square \quad$ Visual onscreen graphic feedback
$\square \quad$ Haptic or touch feedback
$\square \quad$ Custom verbal feedback
$\square \quad$ Customer verbal + haptic/touch feedback

On a scale from $1-5$, where 1 is not at all helpful and 5 is very helpful, how would you rate the helpfulness of having your reflection as feedback?
1
2
3
4
5

not helpful

very helpful

On a scale from $1-5$, where 1 is not at all helpful and 5 is very helpful, how would you rate the helpfulness the visual on screen feedback provided by the gaming system?
1
2
3
4
5
very helpful

not helpful

On a scale from $1-5$, where 1 is not at all helpful and 5 is very helpful, how would you rate the helpfulness and clarity of verbal feedback provided by the instructor?

1

not helpful
23

3
4

very helpful

On a scale from $1-5$, where 1 is not at all helpful and 5 is very helpful, how would you rate the helpfulness haptic/touch feedback provided by the instructor?

$\begin{array}{ccccc}1 & 2 & 3 & 4 & 5 \\ \text { not helpful } & & & & \text { very helpful }\end{array}$


On a scale from 1-5, where 1 is not at all helpful and 5 is very helpful, how would you rate the helpfulness of the combination of haptic/touch feedback and verbal feedback provided by the instructor?

1

not helpful
2

(1)

3

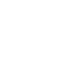
4
5
very helpful 


\section{Appendix D Pose Evaluation Sheets}

\section{D.1 Demonstration DeliveryTest Pose Score Sheet}

\section{TEST 1 - Demonstration Delivery}

Half moon

19

\begin{tabular}{|l|l|}
\hline & Legs straight \\
\hline & Palms touching \\
\hline & Arms inline with ears outstretched above head \\
\hline & Shoulders relaxed - pulled back \\
\hline & Elbows locked \\
\hline & Back straight \\
\hline & Chest open \\
\hline & Hips pushed forward and inline from the side \\
\hline
\end{tabular}

(common difficulty: tilting upper body forward)

Downward dog

19

\begin{tabular}{|l|l|}
\hline & Heels flat on floor \\
\hline & Feet shoulder width apart \\
\hline & Legs straight but knees not locked \\
\hline & Stomach pulled in \\
\hline & Shoulder blades actively pulled back \\
\hline & Neck and head in line with arms \\
\hline & Back arched \\
\hline
\end{tabular}

Warrior 2

$/ 11$

\begin{tabular}{|l|l|}
\hline & Front foot facing front of room \\
\hline & Back foot out at 45 degrees \\
\hline & Front knee bent above ankle \\
\hline & $3.5-4$ foot distance between feet \\
\hline & Thigh as parallel with ground as possible \\
\hline
\end{tabular}




\begin{tabular}{|l|l|}
\hline & Arms up, extended straight \\
\hline & Arms aligned with pelvis \\
\hline & Arms at shoulder height \\
\hline & Palms facing down \\
\hline & Head turned to face forward and Chin aligned with front shoulder \\
\hline
\end{tabular}

\section{Triangle -Section A}

/ 12

\begin{tabular}{|l|l|}
\hline & Front foot pointing towards front of room \\
\hline & Back foot at 90 degree angle \\
& Heels aligned \\
\hline & Legs straight \\
\hline & $3.5-4$ foot distance between feet \\
\hline & Back hand rests on floor on outer side of front foot (can be on foot or calf... but not \\
\hline & Front arm out stretched straight up \\
\hline & Palms facing out away from the body \\
\hline Arms and shoulders in straight line \\
\hline Back straight - not hunched or distorted \\
\hline Gazing up towards the ceiling \\
\hline Chin aligned with shoulder
\end{tabular}

Half spinal twist

19

\begin{tabular}{|l|l|}
\hline & One knee bent with heel close to body \\
\hline & Bent knee touching the ground straight in front of body \\
\hline & Opposite ankle on outside of bent knee \\
\hline & Opposite arm holds ankle over top of opposite knee. \\
\hline & Opposite ankle and opposite knee touching \\
\hline & Borso and head facing side of room \\
\hline & Other hand on floor behind pushing up to help back straighten \\
\hline
\end{tabular}




\section{SECTION B}

Dancer

$/ 13$

\begin{tabular}{|l|l|}
\hline & Standing on one leg \\
\hline & Standing leg straight and knee locked \\
\hline & Same arm stretch forward \\
\hline & Arm straight \\
\hline & Palm facing down - outwards \\
\hline & Opposite hand holding foot from inside \\
\hline & Thumb on sole of foot \\
\hline & Toes pointed on bent leg \\
\hline & Chest forward \\
\hline & Facing forward \\
\hline & Body pulled down as much as possible - pelvis parallel with the floor \\
\hline & Chin touching shoulder - looking down the length of the arm \\
\hline & Shoulders inline straight in front and behind \\
\hline
\end{tabular}

Forward bend

17

\begin{tabular}{|l|l|}
\hline & Hips tucked \\
\hline & Hands on backs of ankles \\
\hline & Chest touching thighs \\
\hline & Face on shins \\
\hline & Straightening legs as much as possible... (knees can be bent) \\
\hline & Back straight \\
\hline & Neck relaxed \\
\hline
\end{tabular}

Warrior 1

$/ 11$

\begin{tabular}{|l|l|}
\hline & Front foot pointing towards front of room \\
\hline & Back foot at 45-degree angle from front foot towards outside of the room \\
\hline & Front knee bent above ankle \\
\hline $3.5-4$ foot distance between feet \\
\hline & Hips facing forward \\
\hline & Arms out stretched straight \\
\hline & Arms in line with ears \\
\hline
\end{tabular}




\begin{tabular}{|l|l|}
\hline & Palms facing each other \\
\hline & Back slightly arched back \\
\hline & Gaze on fingertips \\
\hline
\end{tabular}

Chair Pose

19

\begin{tabular}{|l|l|}
\hline & Feet hip width apart. Parallel. \\
\hline & Knees separated and parallel. \\
\hline & Knees bent - ideally thighs parallel to floor \\
\hline & Arms out stretched straight and parallel. \\
\hline & Palms facing down \\
\hline & Spine and neck straight \\
\hline & Stomach sucked in \\
\hline & Body weight on heels \\
\hline
\end{tabular}

Seated head to knee

\begin{tabular}{|l|l|}
\hline & Seated \\
\hline & One leg outstretched \\
\hline & Other leg bent with foot touching opposite upper thigh \\
\hline & Hands around bottom of outstretched foot \\
\hline & Back can be arched \\
\hline & Forehead touching knee \\
\hline
\end{tabular}

\section{D.2 Feedback Test Pose Score Sheet}


Halfmoon

19

\begin{tabular}{|l|l|}
\hline & Legs straight \\
\hline & Palms touching \\
\hline & Arms inline with ears outstretched above head \\
\hline & Shoulders relaxed - pulled back \\
\hline & Elbows locked \\
\hline & Back straight \\
\hline & Chest open \\
\hline & Hips pushed forward and inline from the side \\
\hline & Body weight on heels \\
\hline
\end{tabular}

Downward Dog

19

\begin{tabular}{|l|l|}
\hline & Heels flat on floor \\
\hline & Feet shoulder width apart \\
\hline & Legs straight but knees not locked \\
\hline & Stomach pulled in \\
\hline & Shoulder blades actively pulled back \\
\hline & Neck and head in line with arms \\
\hline & Back arched \\
\hline & Hips/sit bones pulled back \\
\hline & Heels flat on floor \\
\hline
\end{tabular}

Warrior

$/ 10$

\begin{tabular}{|l|l|}
\hline & Front foot facing front of room \\
\hline & Back foot out at 45 degrees \\
\hline & Front knee bent above ankle \\
\hline & $3.5-4$ foot distance between feet \\
\hline & Thigh as parallel with ground as possible \\
\hline & Arms up, extended straight \\
\hline & Arms aligned with pelvis \\
\hline & Arms at shoulder height \\
\hline & Palms facing down \\
\hline & Head turned to face forward and Chin aligned with front shoulder \\
\hline Back straight \\
\hline
\end{tabular}

Triangle

/ 12

Front foot pointing towards front of room 


\begin{tabular}{|l|l|}
\hline & Back foot at 90 degree angle \\
\hline & Heels aligned \\
\hline & Legs straight \\
\hline & 3.5-4 foot distance between feet \\
\hline & Back hand rests on floor on outer side of front foot or calf \\
\hline & Front arm out stretched straight up \\
\hline & Palms facing out away from the body \\
\hline & Arms and shoulders in straight line \\
\hline & Back straight - not hunched or distorted \\
\hline & Gazing up towards the ceiling \\
\hline & Chin aligned with shoulder \\
\hline
\end{tabular}

\section{Half Spine Twist}

19

\begin{tabular}{|l|l|}
\hline & One knee bent with heel close to body \\
\hline & Bent knee touching the ground straight in front of body \\
\hline & Opposite ankle on outside of bent knee \\
\hline & Opposite arm holds ankle over top of opposite knee. \\
\hline & Opposite ankle and opposite knee touching \\
\hline & Torso and head facing side of room \\
\hline & Both hips touching floor \\
\hline & Other hand on floor behind pushing up to help back straighten \\
\hline
\end{tabular}

